\title{
BEHAVIOR OF MERCURY IN THE FORMIC ACID VENT CONDENSER- FINAL REPORT (U)
}

Author: John R. Zamecnik

Publication Date: July 22, 1996

Keywords: DWPF, Integrated DWPF Melter System, Formic Acid Vent Condenser, Mercury

Retention: Permanent
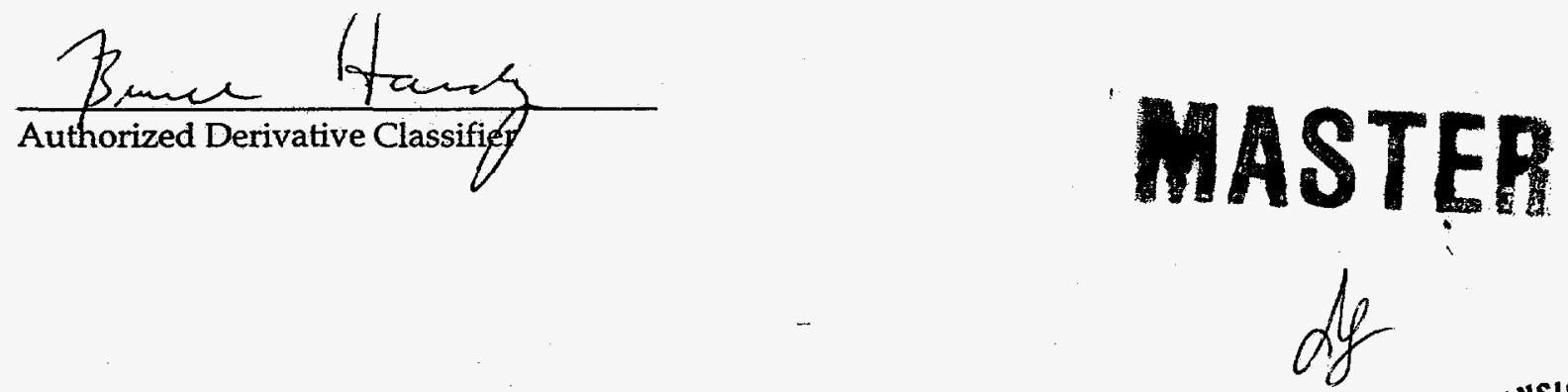

DSTRIBUTION OF THIS DOCUMENT IS UNLIMTTEO

Westinghouse Savannah River Company

Savannah River Site

Aiken, SC 29808

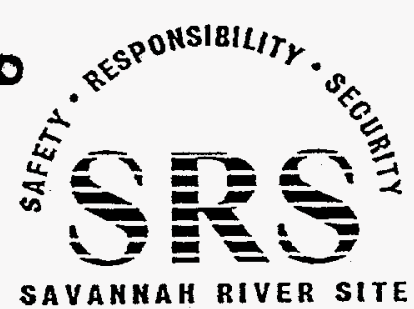

Prepared for the U.S. Department of Energy under contract No. DE-AC09-89SR18035 
WSRC-RP-96-352, Rev. 0

\section{DISTRIBUTION:}

L. M. Papouchado, 773-A

R. A. Jacobs, 704-T

M. N. Brosee, 704-S

S. F. Piccolo, 704-S

R. E. Eibling, 704-T

E. W. Holtzscheiter, 773-A

D. P. Lambert, 704-1T

J. T. Carter, 704-25S

J.C. Marek, 704-T

D. A. Crowley, 773-23A

D. H. Miller, 704-1T

E. F. Duhn, 773-A

P. R. Monson, 704-1T

L. F. Landon, 704-1T

M. E. Smith, 773-23A

C. T. Randall, 704-T

S. R. Young, 773-23A

R. E. Edwards, 704-25S

J. R. Zamecnik, 773-23A

N. E. Bibler, 773-A

J. E. Occhipinti, 704-27S

D. F. Bickford, 773-43A

M. R. Norton, 704-27S

TIM, 703-46A (4) 
WSRC-RP-96-352, Rev. 0

\section{DISCLAIMER}

This report was prepared as an account of work sponsored by an agency of the United States Government. Neither the United States Government nor any agency thereof, nor any of their employees, makes any warranty, express or implied, or assumes any legal liability or responsibility for the accuracy, completeness, or usefulness of any information, apparatus, product, or process disclosed, or represents that its use would not infringe privately owned rights. Reference herein to any specific commercial product, process, or service by trade name, trademark, manufacturer, or otherwise does not necessarily constitute or imply its endorsement, recommendation, or favoring by the United States Government or any agency thereof. The views and opinions of authors expressed herein do not necessarily state or reflect those of the United States Government or any agency thereof.

This report has been reproduced directly from the best available copy.

Available to DOE and DOE contractors from the Office of Scientific and Technical Information, P.O. Box 62, Oak Ridge, TN 37831; prices available from (615) 576-8401.

Available to the public from the National Technical Information Service, U.S. Department of Commerce; 5285 Port Royal Road, Springfield, VA 22161. 


\section{DISCLAMMER}

Portions of this document may be illegible in electronic image products. Images are produced from the best available original document. 


\section{APPROVALS}

Management Approvals:

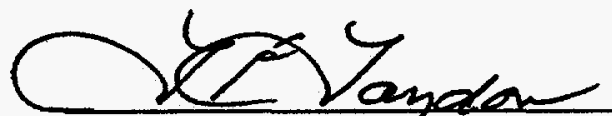

E. W. Holtzschejker, Manager, Defense Waste Processing Technology, SRTC

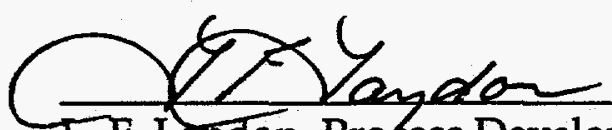

L.F. Landon, Process Development Group, DWPT, SRTC

Technical Review:

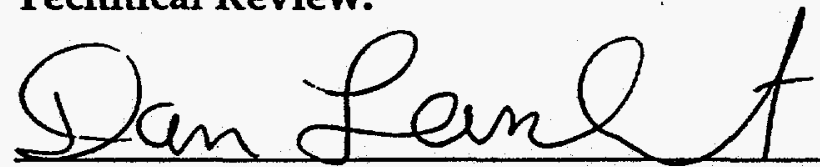

D. P. Lambert, Process Development Group, DWPT, SRTC

Author:

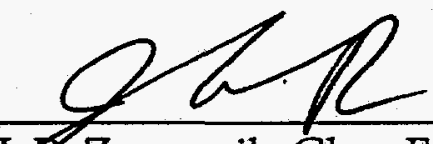

I. J. Zamecnik, Glass Fenmulation and Melter Technology Group, DWPT, SRTC 


\section{TABLE OF CONTENTS}

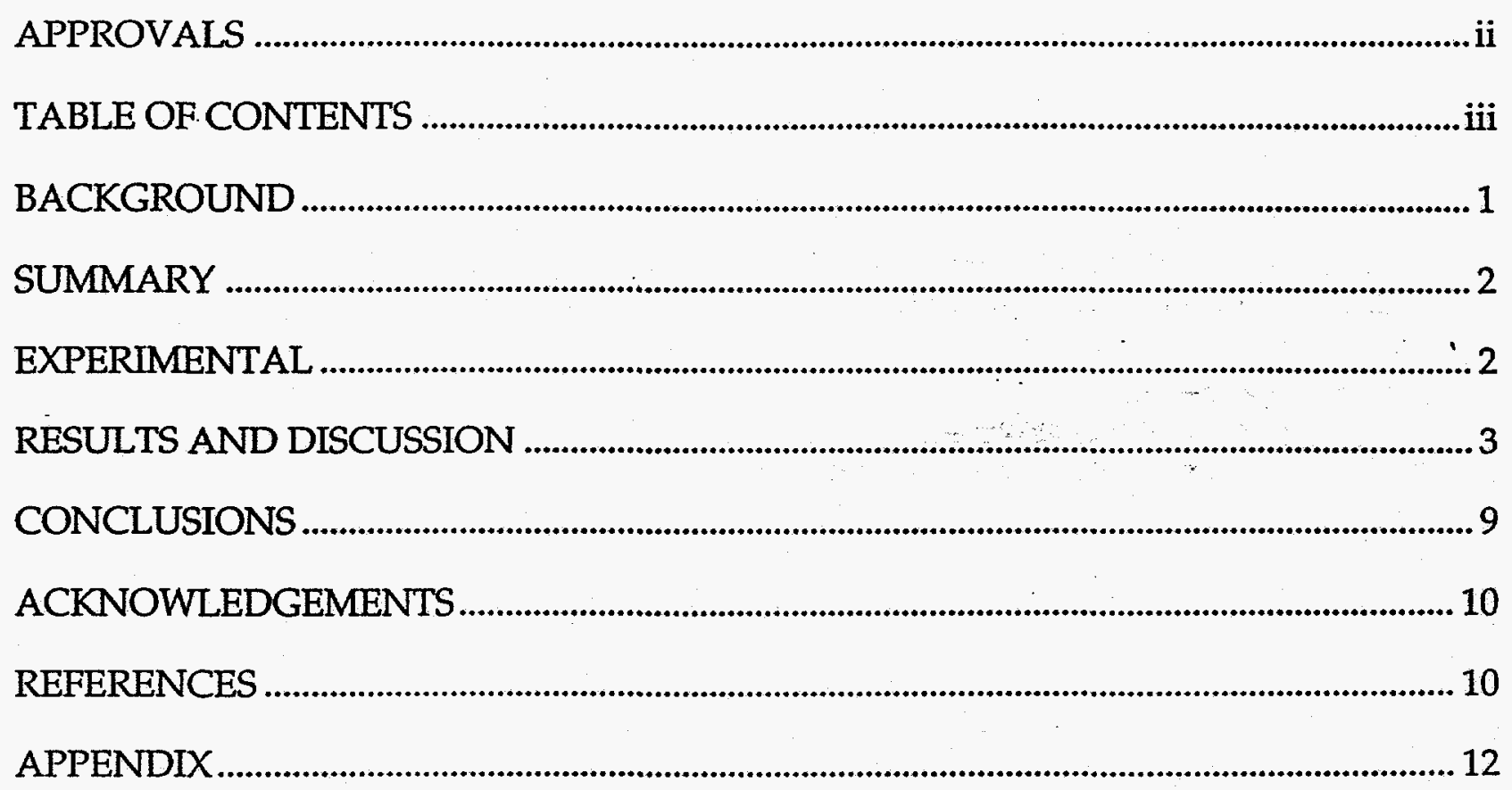




\section{BACKGROUND}

The performance of the Defense Waste Processing Facility (DWPF) Formic Acid Vent Condenser (FAVC) is the subject of a DWPF Technology Issue. Several studies of the performance of this condenser were performed in the Integrated DWPF Melter System (IDMS) at TNX. The FAVC is a chilled water condenser with a High Efficiency Mist Eliminator (HEME) and is designed to cool to $10^{\circ} \mathrm{C}$ the offgas from the Sludge Receipt and Adjustment Tank (SRAT), Slurry Mix Evaporator (SME), Melter Feed Tank (MFT), Recycle Collection Tank (RCT) (all via the Ammonia Scrubbers), and the Precipitate Reactor Bottoms Tank (PRBT). The offgas system is shown in Figure 1 (for IDMS, there is no PRBT and the SRAT is also used as the SME). The scrubbing liquid supply system for the Ammonia Scrubbers is taken from the SMECT, which is maintained at a $\mathrm{pH}$ of 13 by addition of nitric acid. By cooling to $10^{\circ} \mathrm{C}$ or less, water vapor is removed such that it will not condense downstream in the Process Vessel Vent Header, and the mercury content should be reduced to saturation at or below $10^{\circ} \mathrm{C}$. Mercury emissions from DWPF are limited by an environmental permit; ${ }^{1}$ in this permit it is assumed that the mercury concentration exiting the FAVC does not exceed saturation at the exit temperature (which is assumed to be $10^{\circ} \mathrm{C}$ except during peak $\mathrm{NO}_{x}$ evolution).

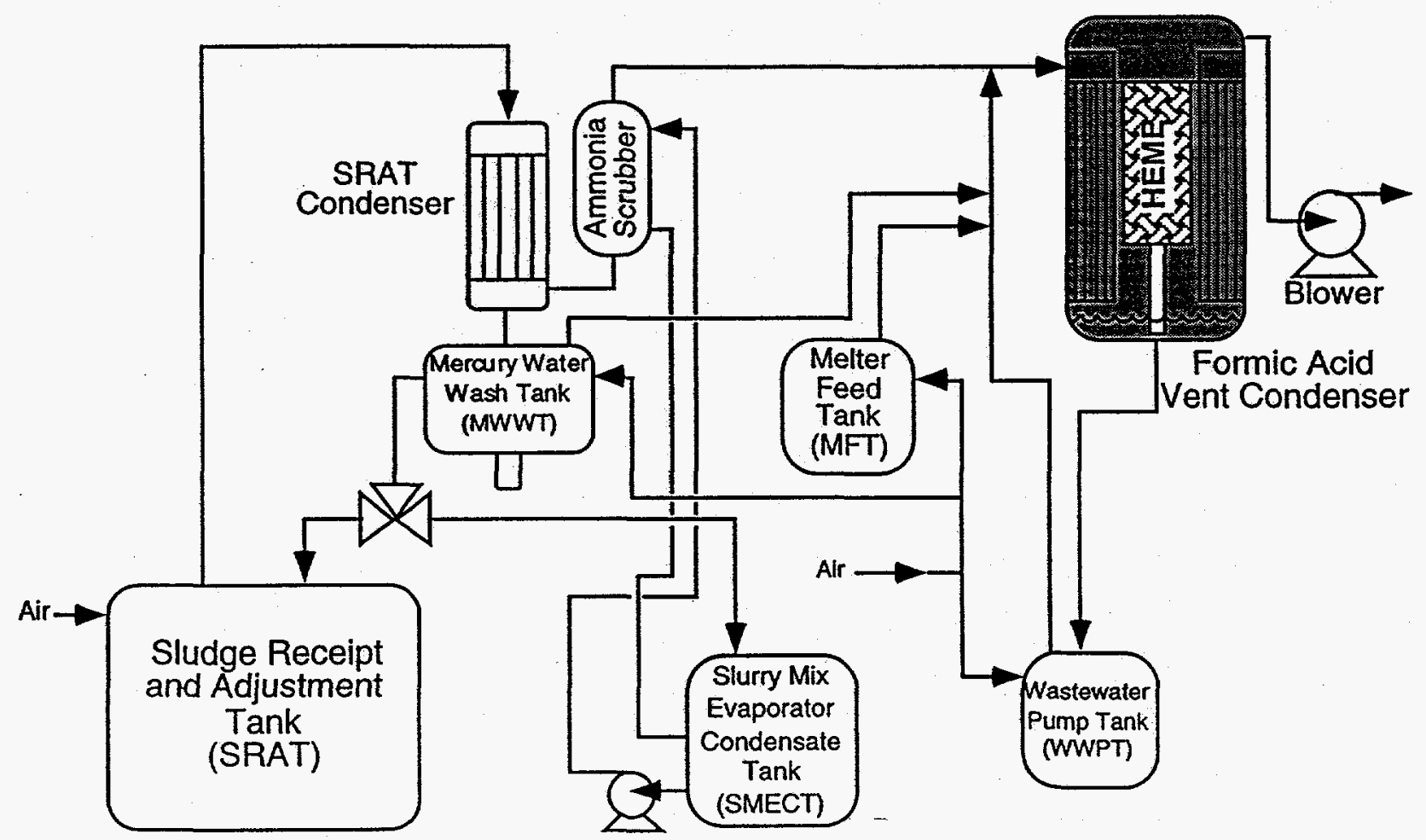

Figure 1. Offgas System Schematic (IDMS).

In the DWPF nitric acid flowsheet, nitric acid is first added to the SRAT at $90-95^{\circ} \mathrm{C}$ to add nitrate and to neutralize basic components in the sludge. No mercury is stripped during this step, since the mercury exists as the oxide in the sludge. Next, Precipitate Hydrolysis Aqueous, or PHA (product from the DWPF Precipitate Hydrolysis Process, 
which contains the cesium removed during the In-Tank Precipitation process), is added to the SRAT at boiling. The PHA contains formic acid and formate that reduce mercuric oxide to elemental mercury; the elemental mercury is then stripped from the SRAT by evaporation during the addition of the PHA. Most of the stripped mercury is condensed in the SRAT condenser and collected in the Mercury Water Wash Tank (MWWT).

To determine if the mercury content of the offgas exiting the FAVC will be at or below saturation, measurements of the mercury concentration in the offgas were performed during the BL1, PX6, and PX7 runs of the Integrated DWPF Melter System (IDMS). Results from the BL1 and PX6 runs have been previously reported. ${ }^{2}$ In these runs, it was found that the concentration of mercury at the FAVC exit substantially exceeded the saturation concentration. For both of these runs, the High Efficiency Mist Eliminator (HEME) in the FAVC was bypassed. In the PX7 run, the HEME was not bypassed, and results from this run are reported herein.

\section{SUMMARY}

The concentrations of mercury at the FAVC inlet and exit were measured during the BL1 and PX6 runs of the Integrated DWPF Melter System (IDMS) with the HEME bypassed and without the ammonia scrubber. The results showed that mercury concentrations of approximately 1.02-12.7 (mean 5.74) times saturation occurred at the FAVC exit. The concentration of mercury at the FAVC inlet was found to be 0.66-6.2 times the saturation value (based on the SRAT condenser exit).

In the PX7 run, the ammonia scrubber was used and the FAVC HEME was not bypassed. The results from this run showed that the FAVC inlet concentrations again were above saturation (1.45-15.5 times saturation), but that the FAVC exit concentrations were only 0.02-0.41 times saturation (except for one data point at 1.61 times saturation).

Operation of the FAVC without the HEME could therefore result in FAVC exit mercury concentrations of greater than 5.74 times saturation, which would result in DWPF emitting greater than $405 \mathrm{lb} / \mathrm{yr}$ of mercury at $100 \%$ attainment; this quantity is well in excess of the permit limit of $175 \mathrm{lb} / \mathrm{yr}$ (for all of DWPF). However, with the HEME in place, the emissions are predicted to be only about $40 \mathrm{lb} / \mathrm{yr}$ for an FAVC exit temperature of $10^{\circ} \mathrm{C}$. The experimental results also indicate that the ammonia scrubbers have little effect on the removal of mercury.

\section{EXPERIMENTAL}

The concentration of total mercury (as $\mathrm{Hg}^{+2}$ and elemental $\mathrm{Hg}$ ) in the offgas was measured by extracting samples from the offgas system. These samples were passed through impingers (bubblers) to absorb the mercury. The gas samples were first passed through two impingers containing $2 \mathrm{M} \mathrm{NaOH}$ to remove most of the $\mathrm{NO}_{\mathrm{x}}$ and protect the downstream impingers from oxidation. The gas samples were then passed through three impingers containing 1.5-4 (w/v)\% $\mathrm{KMnO}_{4}$ in $10(\mathrm{v} / \mathrm{v}) \% \mathrm{H}_{2} \mathrm{SO}_{4}$. The use of 
$\mathrm{KMnO}_{4}$ in $\mathrm{H}_{2} \mathrm{SO}_{4}$ has been described as the most effective method for absorbing elemental mercury, mercury compounds (except organomercury compounds), and particulate mercury. The mercury is oxidized to form $\mathrm{Hg}^{+2}$. A removal efficiency of $90-$ $96 \%$ for a single impinger and $99 \%$ for two or more impingers has been reported. $3,4,5,6,7$

In the presence of oxidizing gases such as $\mathrm{SO}_{2}$ or $\mathrm{NO}_{x}$, pretreatment of the gas sample is necessary to preserve the absorbing ability of the $\mathrm{KMnO}_{4} / \mathrm{H}_{2} \mathrm{SO}_{4}$ absorbent. ${ }^{3}$ For $\mathrm{SO}_{2}$, the use of acidic $\mathrm{H}_{2} \mathrm{O}_{2}$ is recommended. Caustic $(\mathrm{NaOH})$ may also be used, but it will absorb some of the mercury present and convert it to HgO. However, for this work, caustic was used since the impingers containing the caustic were also analyzed for mercury.

The analysis of the impinger samples was performed by Vapor Generation Apparatus (VGA) (cold vapor) atomic absorption (AA) spectroscopy by the DWPT laboratory at TNX. The caustic impinger solutions were treated with acidic permanganate to oxidize all the mercury to $\mathrm{Hg}^{+2}$ and then the mercury was reduced to elemental $\mathrm{Hg}$ by hydroxylamine before measurement on the AA. The $\mathrm{KMnO}_{4} / \mathrm{H}_{2} \mathrm{SO}_{4}$ impinger solutions were also treated with hydroxylamine before measurement on the AA.

\section{RESULTS AND DISCUSSION}

Sampling for mercury at the exit of the FAVC (without the HEME or ammonia scrubber) was conducted during the BL1 (6/90), PX6 (12/93), and PX7 (12/94) runs of the IDMS. In the BL1 and PX7 runs, sampling at the FAVC inlet was also performed. The BL1 run used the DWPF formic acid flowsheet, whereas the PX6 and PX7 runs used the nitric acid flowsheet. Sampling in BL1 was performed during the formic acid reflux period, during which most of the mercury is stripped from the sludge. In PX6 and PX7, sampling was done during the Precipitate Hydrolysis Aqueous (PHA) addition, where most of the mercury is stripped. Sampling during nitric acid addition was not performed since mercury is not evolved during this phase.

Figures 2a-c show the total mercury loading $(\mathrm{mg})$ in each impinger for the samples taken during the three runs (the data are shown on multiple scales so that the values from the start of each train to the end can be seen more clearly). In most cases, the loading of the last $\left(\mathrm{KMnO}_{4}\right)$ impinger was close to zero or much less than the previous impinger. For these samples, virtually all of the mercury was scrubbed in the sample train. However, for some samples, with high mercury loadings in the last impinger, not all of the mercury was scrubbed in the sample train. Therefore, the actual mercury concentrations in the offgas samples were higher than reported. The samples where the mercury would be higher than reported are identified by a $\%$.

The total amount of mercury absorbed by the impinger train was calculated from the analyses of the individual impingers. This information, combined with the volume of gas sampled, was then used to determine the concentration of mercury in the gas sample. 


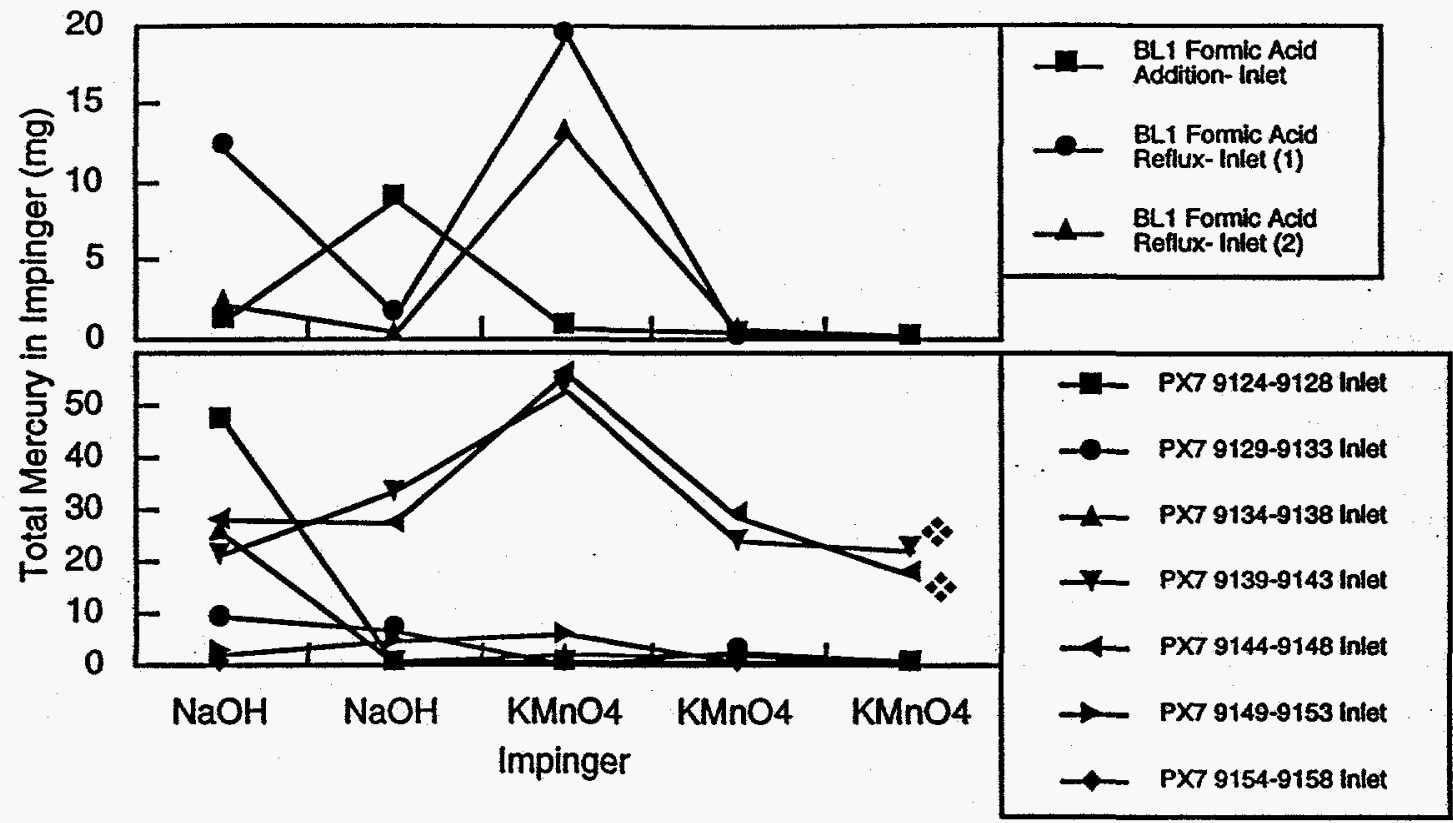

Figure 2a. Total Mercury Loading per Inlet Impinger for the BL1 and PX7 Runs.

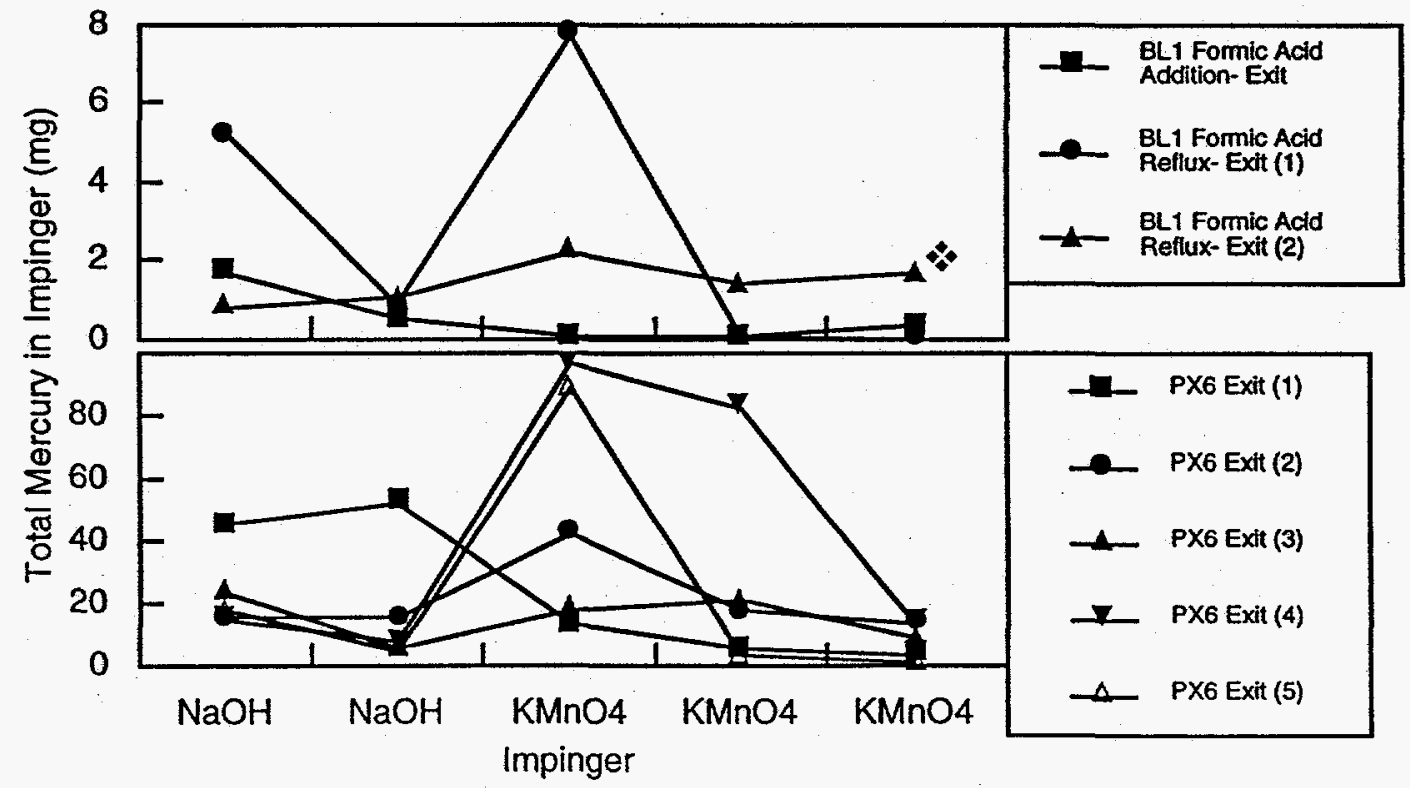

Figure 2b. Total Mercury Loading per Outlet Impinger for the BL1 and PX6 Runs. 
WSRC-RP-96-352, Rev. 0

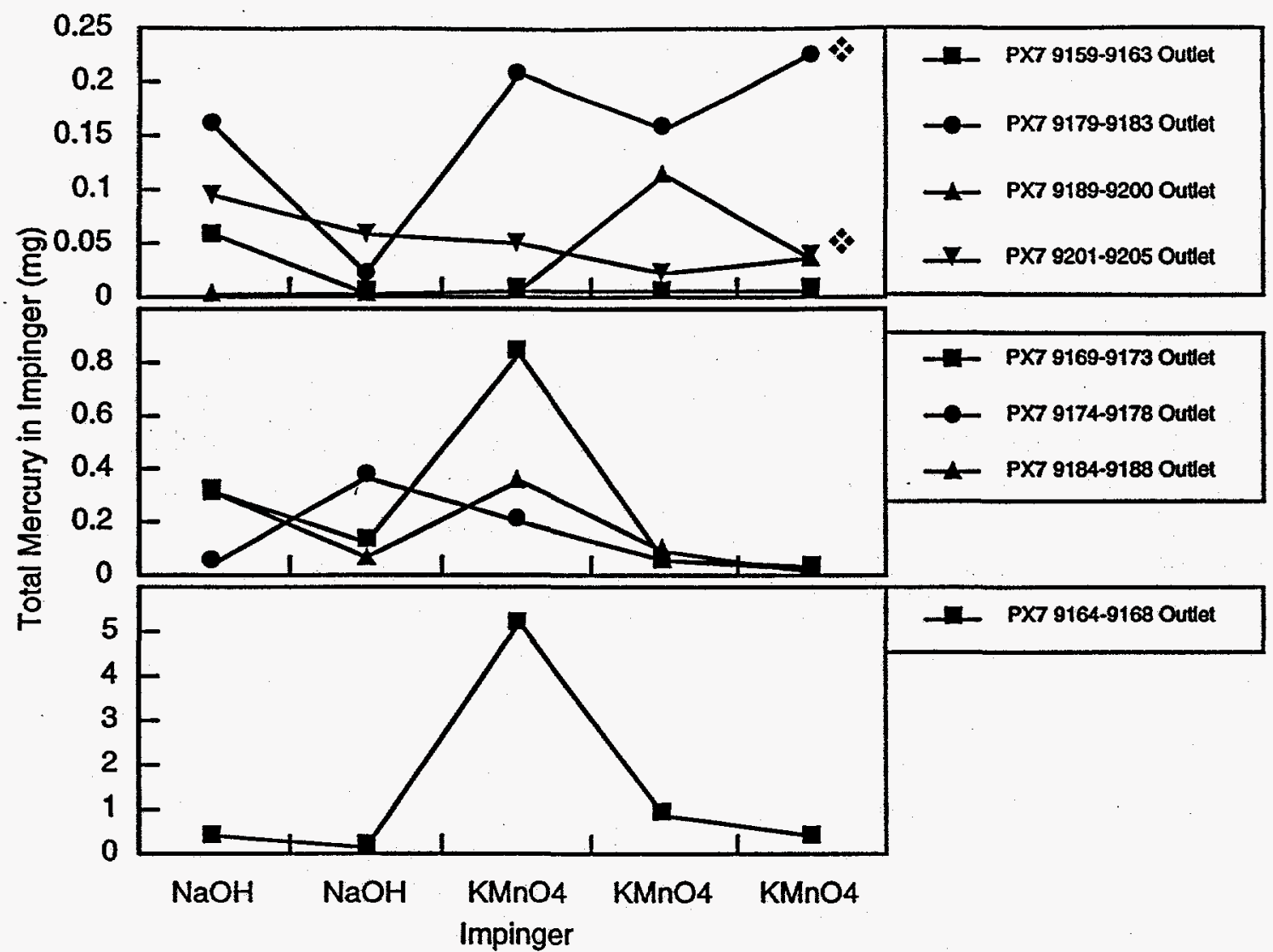

Figure 2c. Total Mercury Loading per Outlet Impinger for the PX7 Run.

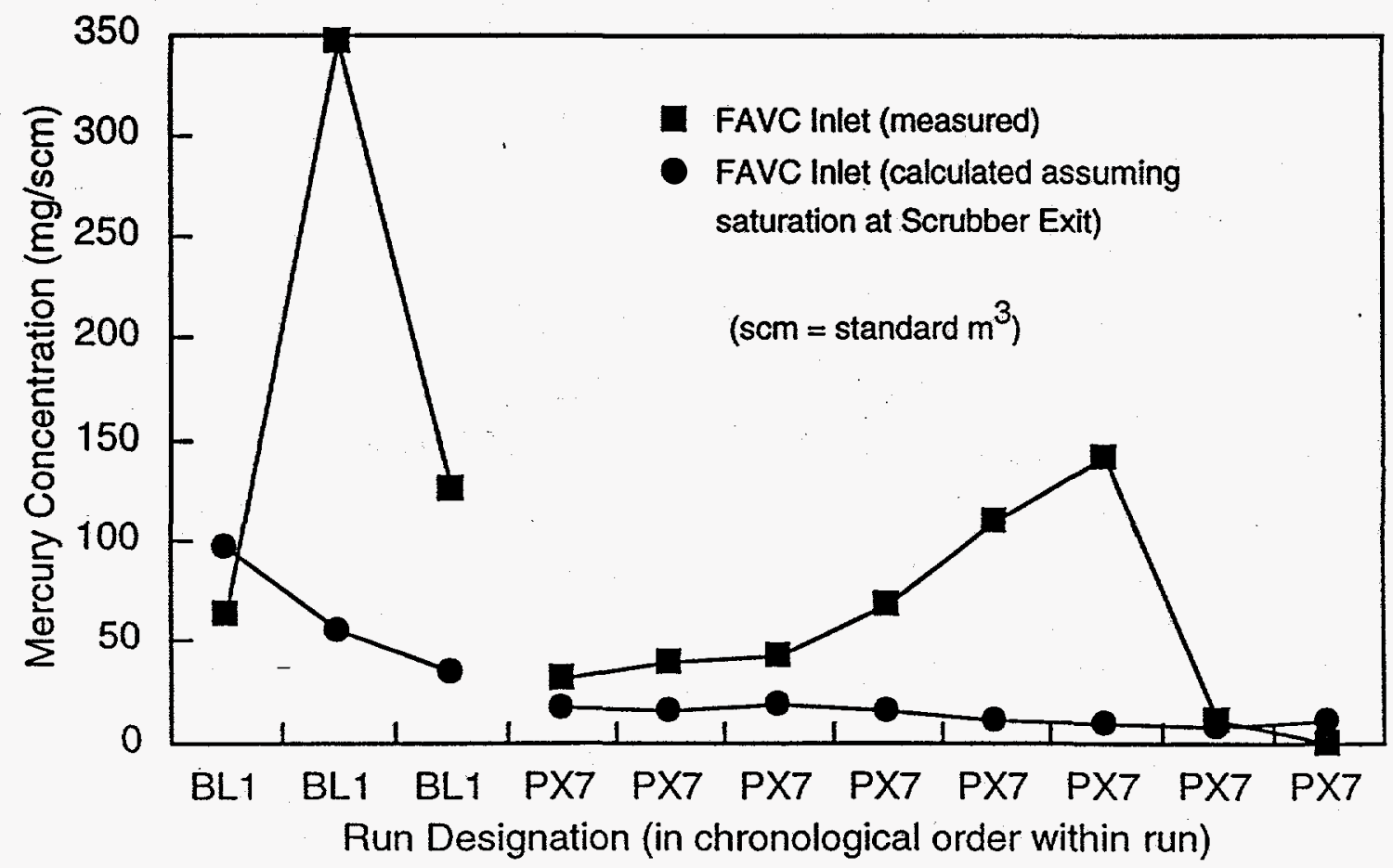

Figure 3. Concentration of Mercury at the FAVC Inlet. 
Figure 3 shows the concentration of mercury measured at the FAVC inlet and the concentration of mercury that would have existed if the offgas had been saturated with mercury at the scrubber exit. The concentration expected at the FAVC inlet is less than that at the scrubber exit due to the dilution by the tank (MWWT, MFT, WWPT) air purges (see Figure 1).

It was expected that the ammonia scrubber would reduce the concentration of mercury in the vapor by stripping since elemental mercury dissolves in nitric acid solutions, forming $\mathrm{Hg}\left(\mathrm{NO}_{3}\right)_{2}$, or mercuric nitrate. However, the concentration of mercury exiting the scrubber was equal to or greater than the saturation concentration at the exit of the SRAT condenser for all but two of the samples. Figure 4 shows the concentration of mercury in the scrubber liquid and the total amount of mercury scrubbed into the scrub solution, both as functions of time. The total amount of mercury increases both from condensation in the SRAT condenser (and dissolution in the condensate) and from scrubbing in the scrubber.

Higher than saturation concentrations could have occurred at the scrubber exit by two mechanisms. The BL1 data can be explained by supersaturation of mercury (or supercooling of the offgas with respect to mercury) at the SRAT condenser exit. Supersaturation is often known as "fogging", where a condensible vapor is condensed to form small droplets or fog in the vapor phase rather than condensing on the condenser tubes into the liquid phase. When fogging occurs, a HEME or other disengagement device is needed to remove the mist. The FAVC has a HEME installed for this very reason. Fogging of mercury at the SRAT condenser exit probably also occurred in the PX7 run, but the scrubber should have removed this fog. However, in PX7, the scrubber vapor exit often contained a fog or entrained scrubber liquid. This entrained liquid or fog would contain mercury at the concentration of the liquid shown in Figure 4 and could account for vapor concentrations greater than saturation.

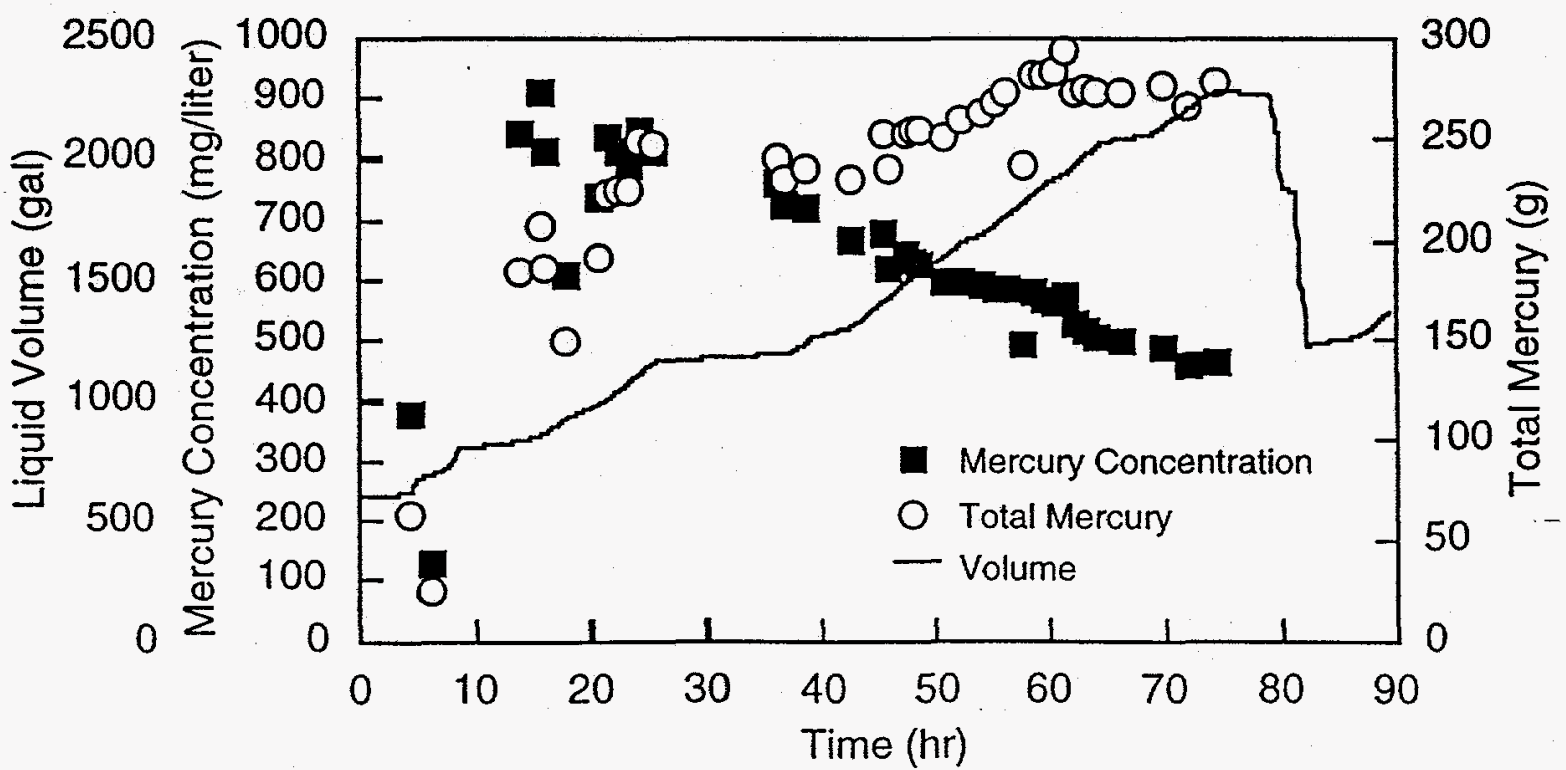

Figure 4. SMECT Mercury Concentration, Total Mercury and Volume of Liquid 


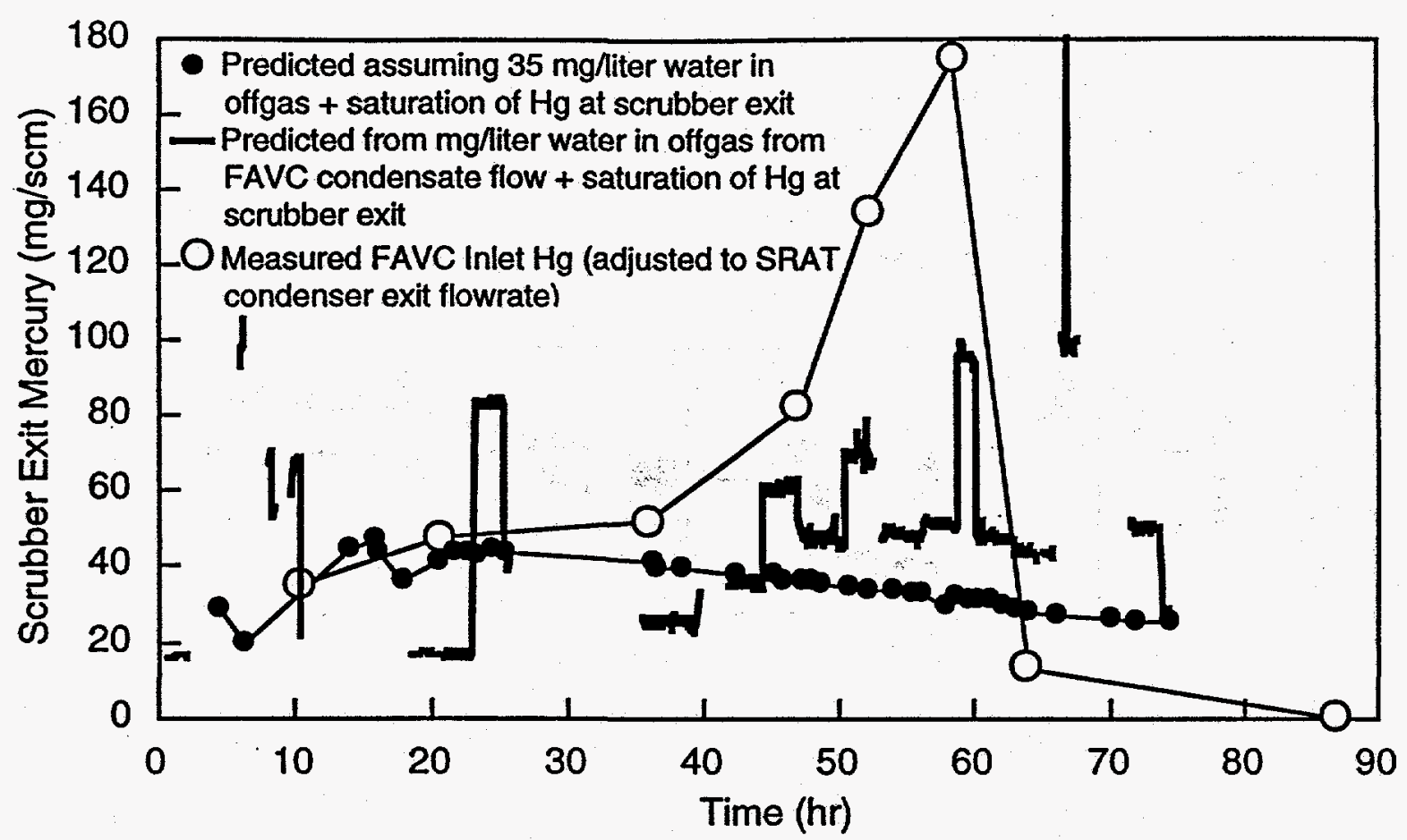

Figure 5. Estimated and Measured Mercury Concentrations at the Scrubber Exit

An estimate of the amount of mercury entrained from the scrubber was made based on limited data (Dräger tube measurements) on the water vapor/fog concentration at the scrubber exit (O in Figure 5). Typical values for water content were $35 \mathrm{mg} / \mathrm{liter}$ of gas. Estimated water concentrations were also made by a material balance around the FAVC (heavy line in Figure 5):

Entrainment $\left.\left.=\begin{array}{cc}\text { Condensate } \\ \text { Flow }\end{array}-\left(\begin{array}{cc}\text { Scrubber Water } & \text { FAVC Exit Water } \\ \text { Vapor Flow } & \text { Vapor Flow } \\ \text { sat'd at Scrubber } \\ \text { Liquid Temp. }\end{array}\right)-\begin{array}{c}\text { sat'd at FAVC } \\ \text { Exit Temp. }\end{array}\right)\right)$

The concentration of mercury in the scrubber exit vapor was assumed to be the amount contained in the entrainment plus the amount of elemental mercury that would exist at saturation at the scrubber exit temperature.

The first three and last points match reasonably well, but the calculated values do not predict the peak seen. Note that the data for the condensate flow was based on the change in the level of the WWPT, which, because of the very low condensate flow, is subject to substantial error. Nonetheless, the data do agree qualitatively with the values calculated assuming $35 \mathrm{mg} /$ liter water in the scrubber exit vapor and do predict higher mercury concentrations near the peaks in the measured data.

Interestingly, the increase in the concentration of mercury exiting the scrubber corresponds almost exactly to the time at which the total amount of mercury in the SMECT liquid levels out (after vertical dotted line in Figure 6), which is also when the 
concentration of mercury in the SMECT begins to drop after the initial increase. Figure 6 also shows that the amount of mercury exiting the SRAT into the offgas system was probably zero during the last two samples (horizontal dotted line), which could help explain the very low values found. (Note: the solid lines are the result of fitting the concentrations as exponentials versus time, but only for data where the SRAT was near boiling ( $\left.\mathrm{T}>90^{\circ} \mathrm{C}\right)$; a plot of these curvefits are shown in the Appendix.)

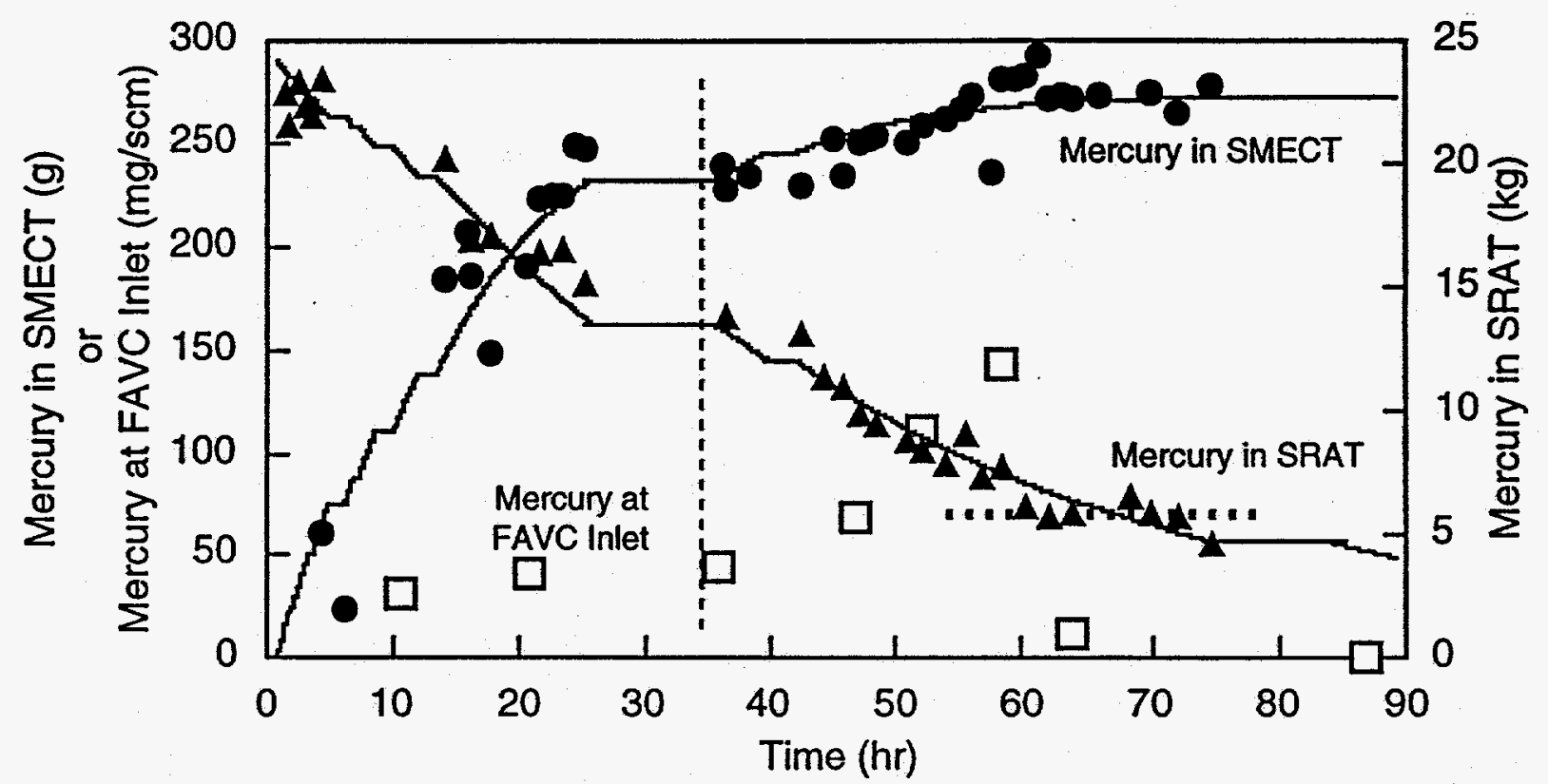

Figure 6. Mercury at the FAVC Inlet, in the SRAT, and in the SMECT

Figure 7 shows the concentration of mercury exiting the FAVC for the BL1 and PX6 runs, without the HEME in line, and the PX7 run with HEME. For the BL1 and PX6 runs, the concentration of mercury leaving the FAVC was substantially above the value at saturation, indicating that fogging occurred. However, for the PX7 run, with the HEME, the concentration of mercury was less than saturation (0.02-0.41 times saturation) for all samples except for one. Even for this one sample above saturation, the concentration was only 1.61 times saturation compared with a mean of 5.74 times saturation for the BL1 and PX6 runs. Concentrations less than saturation were not expected since it should not be possible to reduce the concentration of a vapor below saturation by condensation. A possible reason for the low values could be due to air inleakage into the sample train; however, the EPA Method $111^{7}$ procedures on leak checking were closely followed during the sampling, so air inleakage is very unlikely.

Two possible mechanisms for mercury removal below saturation are proposed. In previous pilot plant runs, the FAVC has been shown to form very concentrated nitric/nitrous acid solutions in the condensate. Acidic condensate on the HEME would be able to dissolve and oxidize (scrub) elemental mercury that condensed, and thus remove it to a concentration below the vapor pressure. 


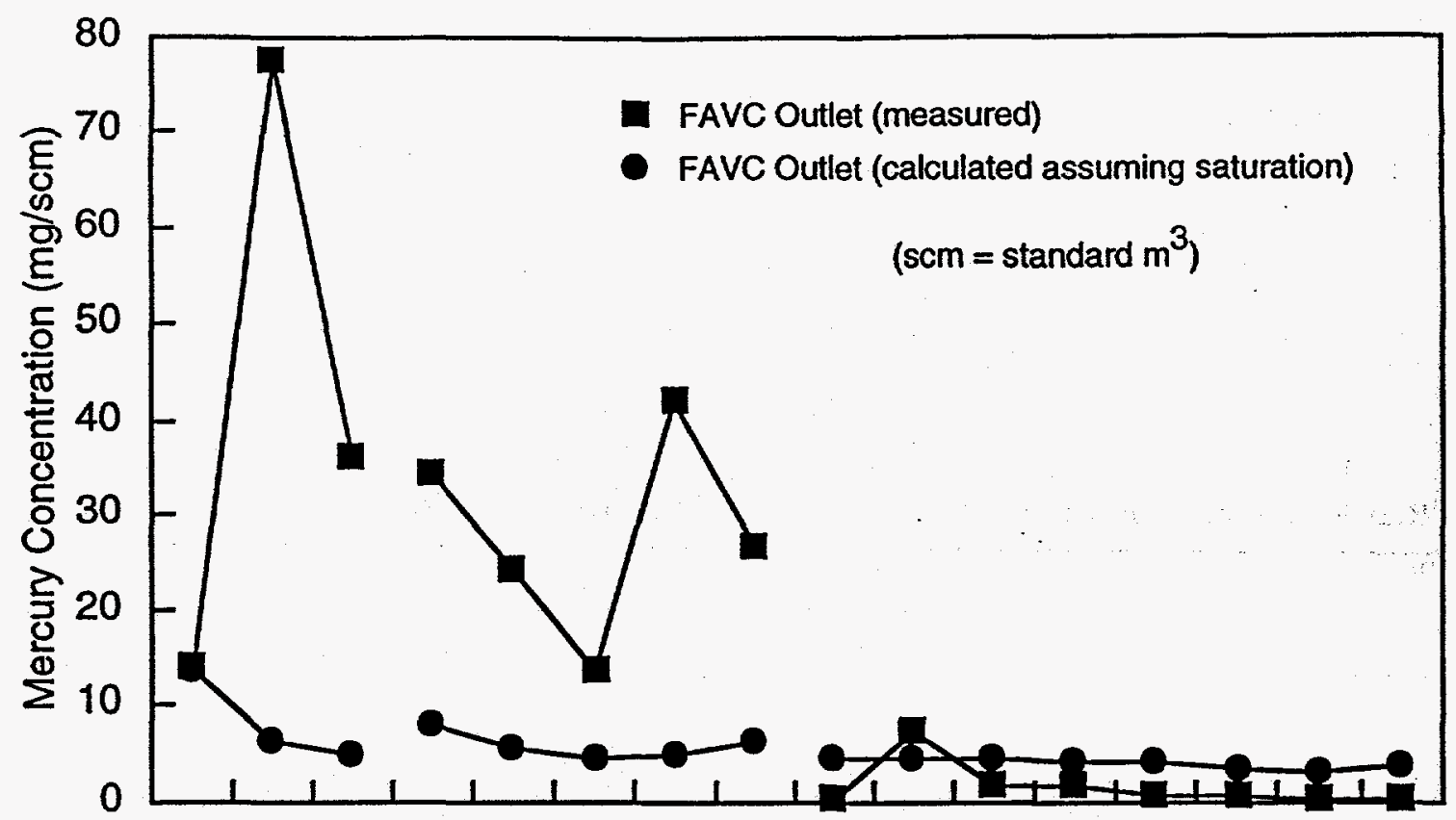

BL1 BL1 BL1 PX6 PX6 PX6 PX6 PX6 PX7 PX7 PX7 PX7 PX7 PX7 PX7 PX7 Run Designation (in chronological order within run)

Figure 7. Concentration of Mercury at the FAVC Exit

The second mechanism is based on the previous discussion of entrainment from the scrubber. If most of the mercury exiting the scrubber was dissolved in the entrained water, it would be easily removed in the FAVC and HEME, since the HEME is designed to efficiently remove entrained droplets. This mechanism seems to be the most likely, but the first may also play a part.

All the concentrations measured at the FAVC inlet and exit for all three runs are summarized in Table I. The DWPF mercury emissions from the FAVC are also shown in this table. The maximum emission would have been $114 \mathrm{lb} / \mathrm{yr}$ for the data point that exceeded saturation; the mean value for all of the data was $24.9 \mathrm{lb} / \mathrm{yr}$ for temperatures from $3-7^{\circ} \mathrm{C}\left(5^{\circ} \mathrm{C}\right.$ mean). The mean value for the FAVC without the HEME was 405 $\mathrm{lb} / \mathrm{yr}$. At the expected operating temperature of $10^{\circ} \mathrm{C}$, the emission rate should be about $40 \mathrm{lb} / \mathrm{yr}$ (calculated as the ratio of vapor pressure at $10^{\circ} \mathrm{C}$ to that at $5^{\circ} \mathrm{C}$ ).

\section{CONCLUSIONS}

The data suggest that the Ammonia Scrubber helps in the removal of mercury, but that entrainment from the scrubber results in a total FAVC inlet mercury concentration approximately equal to what would exist if the scrubber were not present. The entrained mercury is then easily removed in the FAVC and HEME.

Overall, the combination of the Ammonia Scrubber and the Formic Acid Vent Condenser is effective at removing mercury vapor to at or below the saturation concentration at the temperature of the condenser vapor phase, but only with the High 
Efficiency Mist Eliminator (HEME) in place. With the HEME in place, and an operating temperature of $3-7^{\circ} \mathrm{C}$, the emission of mercury from the FAVC in the DWPF should not exceed about $25 \mathrm{lb} / \mathrm{yr}$. For a $10^{\circ} \mathrm{C}$ operating temperature, the emissions should be about $40 \mathrm{lb} / \mathrm{yr}$.

Table I. Summary of Mercury Concentrations

\begin{tabular}{|c|c|c|c|c|c|c|c|c|c|}
\hline Run & $\begin{array}{c}\text { FAVC } \\
\text { Inlet } \\
\text { Meas. } \\
\text { Conc. } \\
\text { (mg/scm) }\end{array}$ & $\begin{array}{c}\text { FAVC Inlet } \\
\text { Conc. (sat. } \\
\text { at Scrubber } \\
\text { Exit) } \\
\text { (mg/scm) }\end{array}$ & $\begin{array}{l}\text { FAVC Inlet } \\
\% \text { Saturation }\end{array}$ & $\begin{array}{c}\text { FAVC } \\
\text { Exit Meas. } \\
\text { Conc. } \\
(\mathrm{mg} / \mathrm{scm})\end{array}$ & $\begin{array}{c}\text { FAVC } \\
\text { Exit Sat. } \\
\text { Conc. } \\
(\mathrm{mg} / \mathrm{scm})\end{array}$ & $\begin{array}{l}\text { FAVC Exit } \\
\% \text { Saturation }\end{array}$ & $\begin{array}{l}\text { FAVC } \\
\text { Inlet } \\
\text { Temp. } \\
\left({ }^{\circ} \mathrm{C}\right)\end{array}$ & $\begin{array}{l}\text { FAVC } \\
\text { Exit* } \\
\text { Temp. } \\
\left({ }^{\circ} \mathrm{C}\right)\end{array}$ & $\begin{array}{c}\text { Estimated } \\
\text { DWPF Hg } \\
\text { Emission } \\
\text { (lb/yr) }\end{array}$ \\
\hline BLI & $\begin{array}{l}63.3 \\
346 . \\
125 .\end{array}$ & $\begin{array}{l}96.4 \\
56.0 \\
34.8\end{array}$ & $\begin{array}{r}66 . \\
618 . \\
359 .\end{array}$ & $\begin{array}{l}14.0 \\
77.5 \\
36.0\end{array}$ & $\begin{array}{r}13.7 \\
6.09 \\
4.90\end{array}$ & $\begin{array}{r}102 . \\
1270 . \\
735 .\end{array}$ & & $\begin{array}{r}20.5 \\
11.1 \\
8.7\end{array}$ & $\begin{array}{r}72 . \\
896 . \\
519 .\end{array}$ \\
\hline PX6 & & & & $\begin{array}{l}34.3 \\
24.0 \\
13.4 \\
42.0 \\
26.5\end{array}$ & $\begin{array}{l}7.96 \\
5.54 \\
4.50 \\
4.69 \\
6.27 \\
\end{array}$ & $\begin{array}{l}432 . \\
434 . \\
298 . \\
895 . \\
423 .\end{array}$ & & $\begin{array}{r}13.6 \\
9.6 \\
7.4 \\
7.9 \\
11.0 \\
\end{array}$ & $\begin{array}{l}305 . \\
306 . \\
210 . \\
632 . \\
299 .\end{array}$ \\
\hline & & Mean & 348. & & Mean & 574. & & Mean & 405. \\
\hline PX7 & $\begin{array}{c}31.3 \\
39.9 \\
42.8 \\
67.5 \\
110 . \\
142 . \\
10.8 \\
0.1 \\
\end{array}$ & \begin{tabular}{r|}
17.3 \\
15.8 \\
17.9 \\
15.5 \\
11.1 \\
9.2 \\
7.5 \\
10.9 \\
\end{tabular} & $\begin{array}{r}181 . \\
253 . \\
239 . \\
435 . \\
990 . \\
1550 . \\
145 . \\
1 .\end{array}$ & $\begin{array}{l}0.082 \\
7.10 \\
1.70 \\
1.73 \\
0.440 \\
0.590 \\
0.108 \\
0.197\end{array}$ & \begin{tabular}{l|}
4.28 \\
4.41 \\
4.36 \\
4.24 \\
3.93 \\
3.24 \\
2.94 \\
3.60
\end{tabular} & \begin{tabular}{r|}
1.9 \\
161. \\
38.9 \\
40.9 \\
11.2 \\
18.2 \\
3.7 \\
5.5
\end{tabular} & $\begin{array}{l}21.2 \\
20.5 \\
21.5 \\
20.0 \\
19.2 \\
17.2 \\
15.8 \\
16.5\end{array}$ & $\begin{array}{l}6.9 \\
7.2 \\
7.1 \\
6.8 \\
6.0 \\
4.0 \\
3.0 \\
5.1\end{array}$ & $\begin{array}{c}1.34 \\
114 . \\
27.5 \\
28.9 \\
7.91 \\
12.8 \\
2.61 \\
3.88\end{array}$ \\
\hline & & Mean & 474. & & Mean & 35. & & Mean & 24.9 \\
\hline
\end{tabular}

* FAVC exit temperature calculated as shown in the Appendix ${ }^{8}$

\section{ACKNOWLEDGEMENTS}

The author would like to acknowledge the hard work of Frances Williams and Nick Odom in performing the sampling during these runs. The technical review and input of Dan Lambert, Russ Eibling, and Jim Marek is also greatly appreciated.

\section{REFERENCES}

1. Jacobs, R. A., Bounding Estimate of DWPF Mercury Emissions, WSRC-RP-92-1205, Rev. 2, November 15, 1993.

2. Zamecnik, J. R., Behavior of Mercury in the Formic Acid Vent Condenser-Interim Report, WSRC-RP-94-1141, Rev. 0, October 11, 1994. 
3. Driscoll, J. N., Sampling and Analytical Techniques for Mercury in Stationary Sources: A State-of-the-Art Report,"Health Lab. Sci., 11(4), p. 348 (1974).

4. McQuaker, N. R., Sandberg, D. K., The Determination of Mercury Source Emissions in the Presence of High Levels of $\mathrm{SO}_{2}, \mathrm{~J}$. Air Poll. Cont. Assoc., 32(6), p. 634 (1982).

5. Makris, W. E., Crawford, C. J., Miller, R. O., Bell, Z. G., Jr., The Comparison of Five Published Methods for the Determination of Mercury-In-Air, Jt. Conf. Sens. Environ. Pollut., (Conf. Proc., 4th), p. 192 (1978).

6. Shendrikar, A. D., Briden, F. E., Mercury Atmosphere Generation and Media Collection Efficiency Evaluations for the SASS Impinger System, Specialty Conf. on: Measurement and Monitoring of Non-Criteria (Toxic) Contaminants in Air, Frederick, E. R. (ed.), Chicago IL, Mar. 22-24, 1983.

7. Method 111. Determination of Particulate and Gaseous Mercury Emissions From Sewage Sludge Incinerators, Federal Register, Doc. 80-31822, October 15, 1980.

8. Zamecnik, J. R., The Effect of Nitric Oxide Oxidation on the DWPF Formic Acid Vent Condenser (FAVC) Exit Temperature (U), WSRC-RP-92-1174, October 29, 1992. 


\section{APPENDIX}

WSRC-TR-96-352, Rev. 0

\section{SUMMARY OF PX7 MERCURY RESULTS}

Table. PX7 Summary

$\begin{array}{rlcccc}\text { Date } & \text { Time } & (\mathrm{mg} / \mathrm{scm}) & (\mathrm{mg} / \mathrm{scm}) & \text { in FAVC } & \text { \% Saturation } \\ 12 / 9-10 / 94 & 19: 05-07: 50 & 31.3 & 0.082 & 99.7 & 1.9 \\ 12 / 10 / 94 & 08: 15-15: 15 & 39.9 & 7.10 & 82.2 & 161 . \\ 12 / 10-11 / 94 & 15: 30-10: 30 & 42.8 & 1.70 & 96.0 & 38.9 \\ 12 / 11 / 94 & 11: 35-16: 20 & 67.5 & 1.73 & 97.4 & 40.9 \\ 12 / 11 / 94 & 16: 45-21: 45 & 110 . & 0.440 & 99.6 & 11.2 \\ 12 / 11-12 / 94 & 23: 00-04: 00 & 142 . & 0.59 & 99.6 & 18.2 \\ 12 / 12 / 94 & 04: 14-09: 45 & 10.8 & 0.108 & 99.0 & 3.7 \\ 12 / 13 / 94 & 03: 30-08: 30 & 0.076 & 0.197 & -228 . & 5.5\end{array}$

\section{CURVEFITS OF MERCURY IN SRAT AND SMECT}

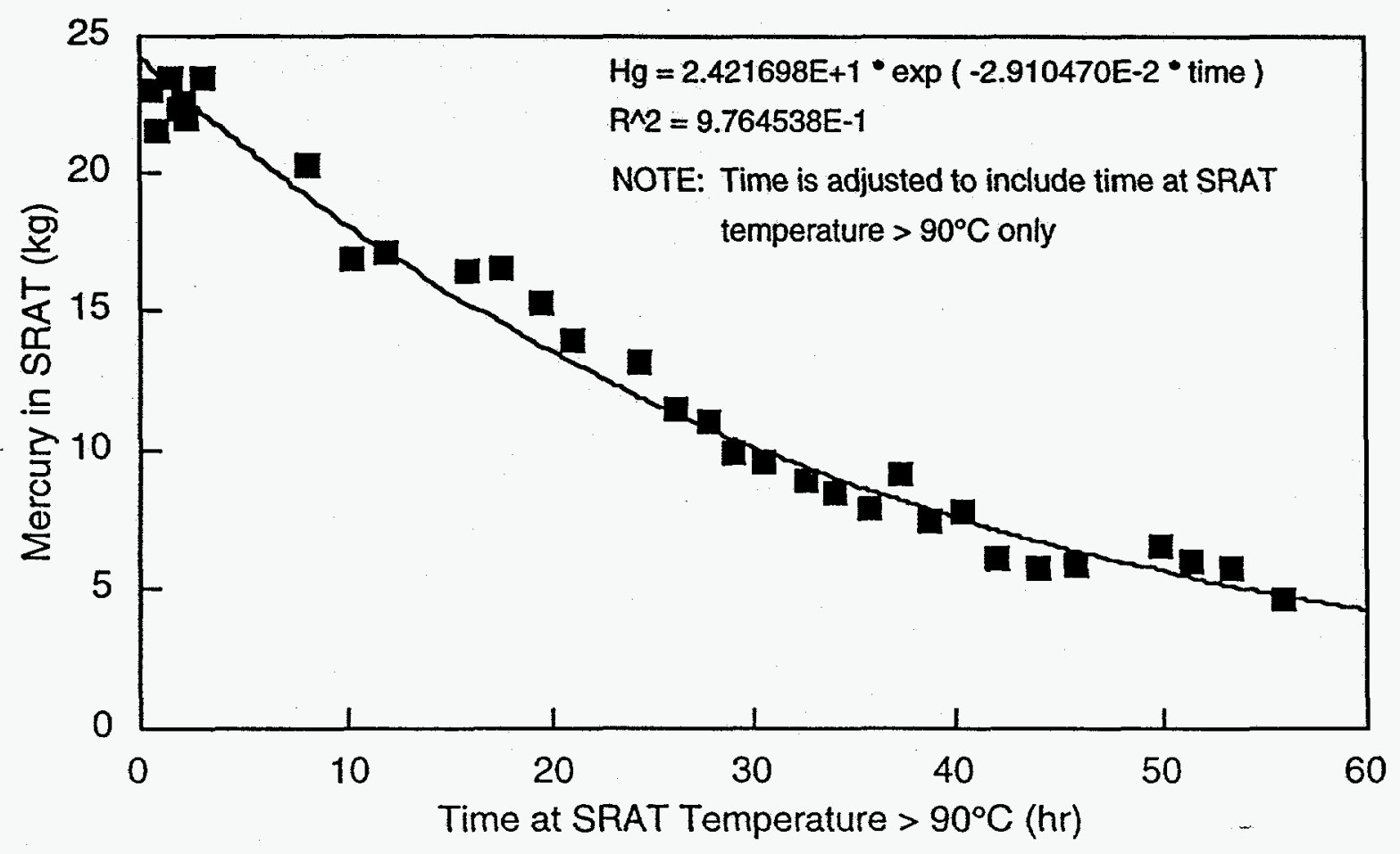




\section{APPENDIX}

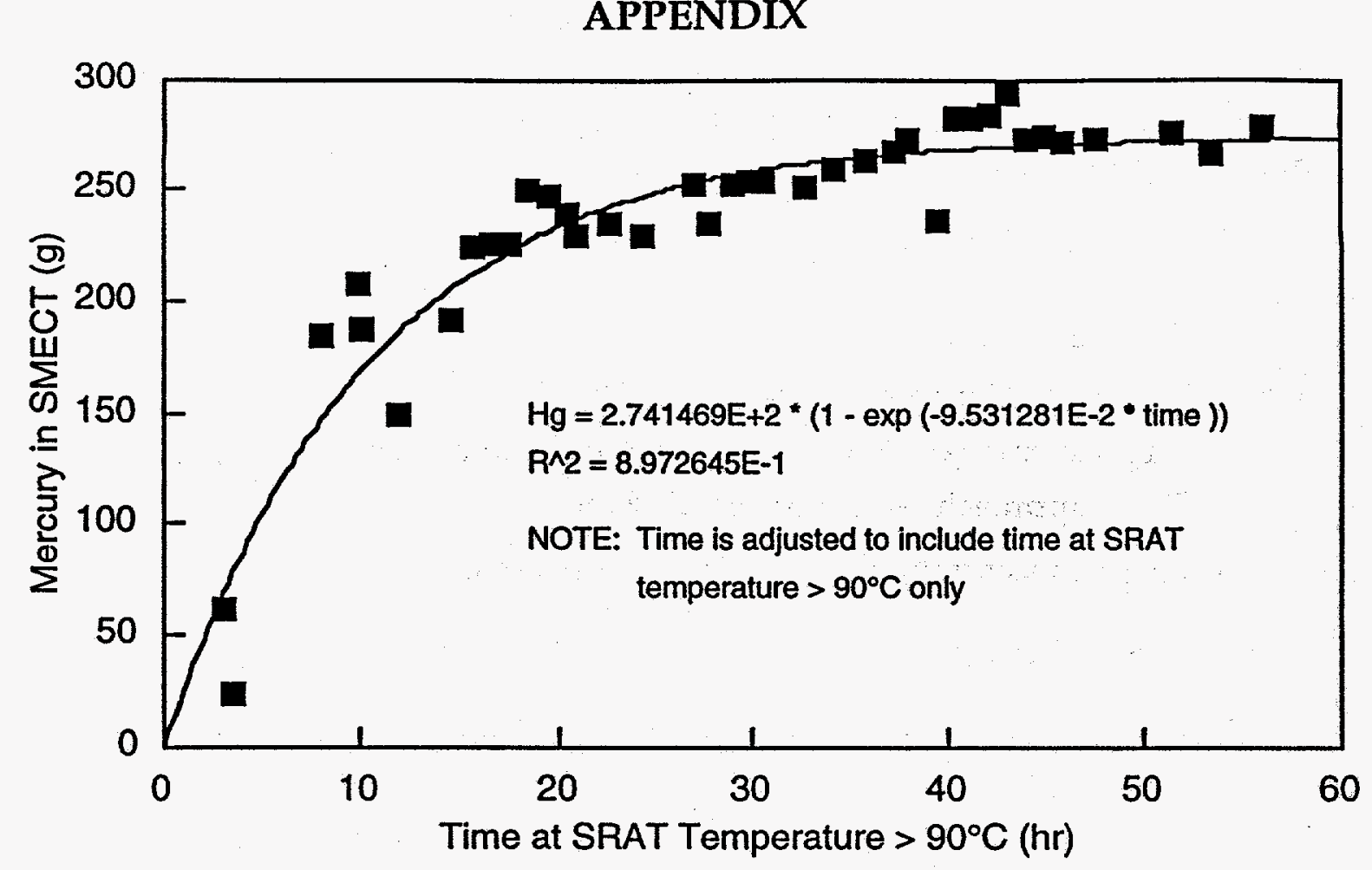

\section{SATURATION VAPOR PRESSURE OF MERCURY}

$$
\mathrm{P}_{\mathrm{Hg}}^{\text {sat }}=\exp \left(18.072-\frac{7079.256}{\mathrm{~T}+265.47}\right)
$$

where $\mathrm{p}_{\mathrm{Hg}}^{\text {sat }}=$ torr

$$
\mathrm{T} \equiv{ }^{\circ} \mathrm{C}
$$

\section{ESTIMATION OF FAVC TUBE EXIT TEMPERATURE}

For the BL1 data, the FAVC HEME exit temperature was calculated by using models developed to predict the temperature rise in the FAVC due to NO oxidation (see reference 8 ):

$$
\mathrm{T}_{\text {HEME }}=2.9171\left[\mathrm{NO}_{x}\right]^{0.5681}+3.7210 \beta\left[\mathrm{NO}_{\mathrm{x}}\right]^{0.3331}+\mathrm{T}_{\mathrm{amb}}+\mathrm{T}_{\mathrm{c}}+\mathrm{T}_{\text {app }}
$$

where $\mathrm{T}_{\text {HEME }}=$ FAVC HEME exit temperature $\left({ }^{\circ} \mathrm{C}\right)$

$$
\mathrm{T}_{\mathbf{c}}=\text { average cooling water temperature (inlet \& outlet) }\left({ }^{\circ} \mathrm{C}\right)
$$

$\mathrm{T}_{\text {app }}=$ approach temperature $\left({ }^{\circ} \mathrm{C}\right) \approx 1.6$

$\mathrm{T}_{\mathrm{amb}}=$ temperature rise from tube exit to HEME exit due to ambient heat conduction $\left({ }^{\circ} \mathrm{C}\right.$ $\left[\mathrm{NO}_{\mathrm{x}}\right]=$ concentration of $\mathrm{NO}_{\mathrm{x}}$ at FAVC exit (mol \%)

$\beta=$ bypassing factor (= 0 if HEME bypassed; $=1$ if HEME not bypassed) 


\section{APPENDIX}

WSRC-TR-96-352, Rev. 0

The predicted and measured FAVC HEME exit temperatures are shown in Figure A-1. Since the data fit well, estimation of the tube exit temperature using the models was deemed appropriate.

The FAVC tube exit temperature was estimated as:

$$
\mathrm{T}_{\text {tube }}=2.9171\left[\mathrm{NO}_{\mathrm{x}}\right]^{0.5681}+\mathrm{T}_{\mathrm{c}}+\mathrm{T}_{\text {app }}
$$

where $\mathrm{T}_{\text {tube }}=\mathrm{FAVC}$ tube exit temperature $\left({ }^{\circ} \mathrm{C}\right)$

$\mathrm{T}_{\mathrm{c}}=$ average cooling water temperature (inlet \& outlet) $\left({ }^{\circ} \mathrm{C}\right)$

$\mathrm{T}_{\text {app }}=$ approach temperature $\left({ }^{\circ} \mathrm{C}\right)=1.6$

$\left[\mathrm{NO}_{\mathrm{x}}\right]=$ concentration of $\mathrm{NO}_{\mathrm{x}}$ at FAVC exit (mol \%)

The predicted FAVC tube exit temperature is also shown in Figure A-1.

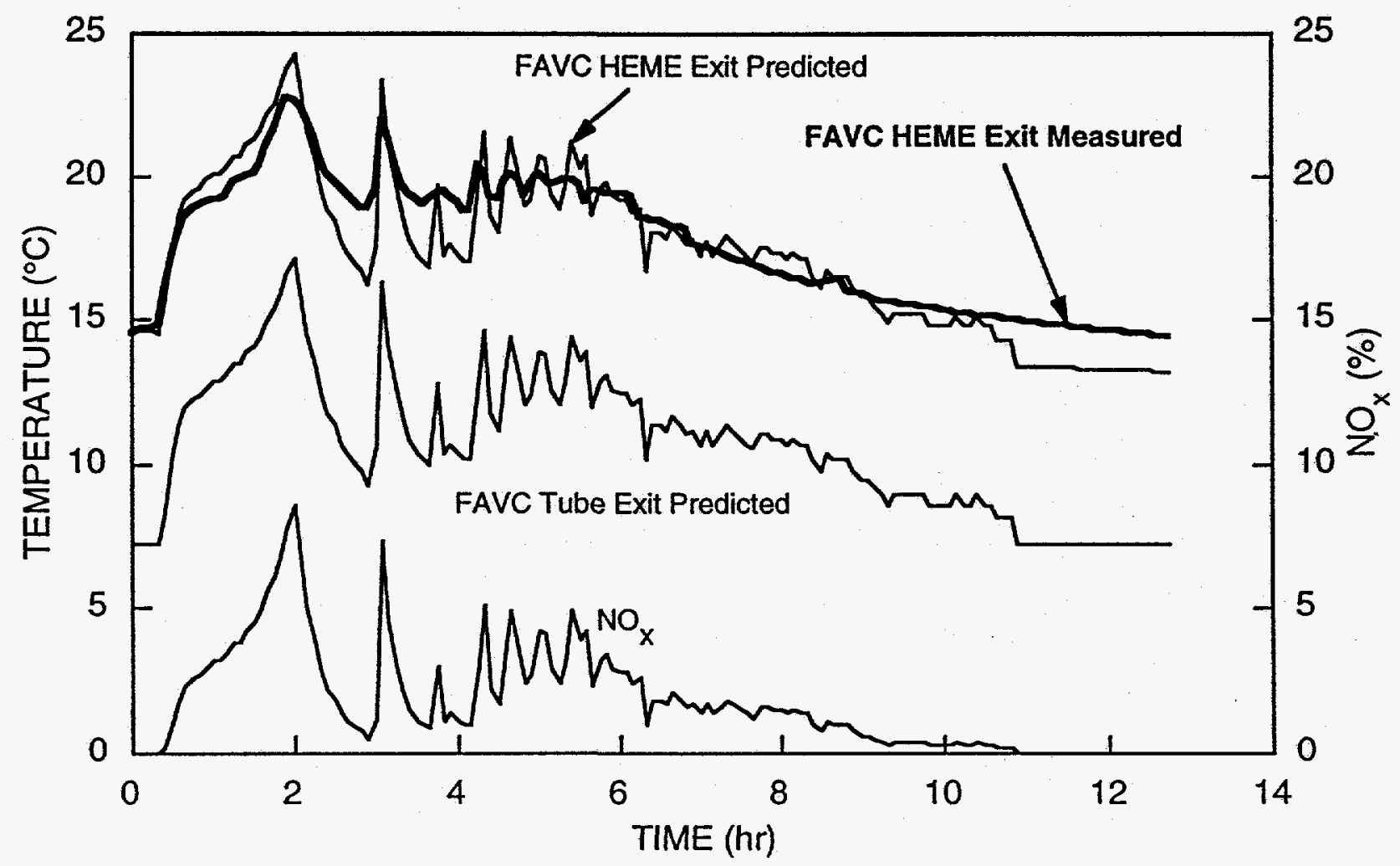

Figure A-1. Measured and Predicted FAVC Temperatures for IDMS Run BL1

\section{ANALYSIS OF MERCURY SAMPLING DATA}

See attached data analysis sheets. 


\section{APPENDIX}

WSRC-TR-96-352, Rev. 0

IDMS RUN: PUREX 7

COMMENTS PX7 9119-9123 Inlet

DATE: $12 / 9-10 / 1994$

TIME: 19:05-20:15, 21:30-22:00, 04:50-07:50

WASHED SAMPLE:

\begin{tabular}{|c|c|c|c|c|c|c|}
\hline \multirow{3}{*}{\multicolumn{2}{|c|}{ UPLE: }} & \multicolumn{2}{|c|}{ IDMS \# ID-RUN-IMPI } & & & \multirow[b]{2}{*}{9123} \\
\hline & & 9119 & 9120 & 9121 & 9122 & \\
\hline & & $\mathrm{NaOH}$ & $\mathrm{NaOH}$ & $\mathrm{KMnO4}$ & $\mathrm{KMnO4}$ & $\mathrm{KMnO} 4$ \\
\hline Empty bottle & g & 37.96 & 38.18 & 37.98 & 38.31 & 38.47 \\
\hline Sample + wash water & $\mathrm{g}$ & 157.19 & 191.61 & 418.76 & 326.10 & 152.47 \\
\hline Weight of sample & g & 119.23 & 153.43 & 380.78 & 287.79 & 114.00 \\
\hline Approx. density of sample & $\mathrm{g} / \mathrm{m}$ & 1.10 & 1.10 & 1.10 & 1.10 & 1.10 \\
\hline Volume of sample & $\mathrm{m}$ & 108.4 & 139.5 & 346.2 & .261 .6 & 103.6 \\
\hline Conc of $\mathrm{Hg}$ & mg/iter & 133.0 & 20.4 & 15.6 & 1.9 & 0.3 \\
\hline $\mathrm{Hg}$ collected & $\mathbf{m g}$ & 14.4 & 2.8 & 5.4 & 0.5 & 0.0 \\
\hline$\%$ of total collected & $\%$ & 62.17 & 12.27 & 23.29 & 2.14 & 0.12 \\
\hline
\end{tabular}

Total Hg collected $\quad \mathrm{mg} / 23.2$

DRY GAS SAMPLE: Gas Volume cuft 28.60

MERCURY CONCENTRATION MEASURED AT FAVC INLET:

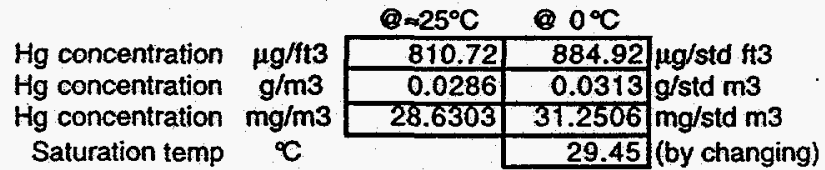

MERCURY CONCENTRATION AT SRATC EXIT CALCULATED FROM MEASURED FAVC INLET:

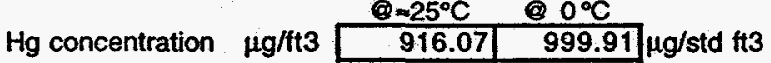

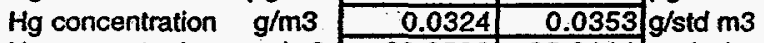

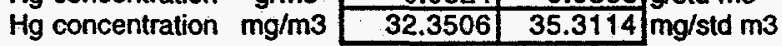

MERCURY CONCENTRATION AT SATURATION (PROCESS BASIS, SRATC EXIT) : Average SRATC Exit Temperature $\subset$ 23.80

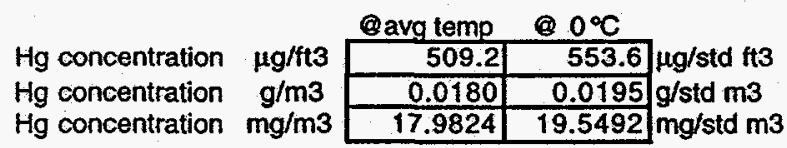

MERCURY CONCENTRATION AT FAVC INLET CALCULATED FROM SATURATION:

SRAT/FAVC flow ratio

FAVC inlet Temperature

$\varnothing \quad 0.885$

$\mathrm{Hg}$ concentration $\mu \mathrm{g} / \mathrm{ft} 3$

Hg concentration $\mathrm{g} / \mathrm{m} 3$

QFAVC In T $00^{\circ} \mathrm{C}$

$\mathrm{Hg}$ concentration $\mathrm{mg} / \mathrm{m} 3$

\begin{tabular}{r|r}
454.6 & 489.9
\end{tabular}$\mu \mathrm{g} / \mathrm{std} \mathrm{ft3}$

\begin{tabular}{r|r|r|}
\hline 0.0161 & 0.0173 & $\mathrm{~g} / \mathrm{std} \mathrm{m3}$ \\
\hline
\end{tabular}

\begin{tabular}{lll}
\hline 16.0550 & 17.3011 & $\mathrm{mg} / \mathrm{std} \mathrm{m} 3$ \\
\hline
\end{tabular}

MEASURED / EXPECTED @ SATURATION

1.806 


\section{APPENDIX}

IDMS RUN: PUREX 7

COMMENTS PX7 9124-9128 Inlet

DATE: $12 / 10 / 94$

TIME: 08:15-15:15

WASHED SAMPLE:

\begin{tabular}{|c|c|c|c|c|c|c|}
\hline \multirow{3}{*}{\multicolumn{2}{|c|}{ E: }} & \multicolumn{5}{|c|}{ IDMS \# IDARUN-MPI- } \\
\hline & & 9124 & 9125 & 9126 & 9127 & 9128 \\
\hline & & $\mathrm{NaOH}$ & $\mathrm{NaOH}$ & KMnO4 & KMnO4 & KMnO4 \\
\hline Empty bottle & g] & 38.04 & 37.95 & 38.45 & 38.76 & 38.01 \\
\hline Sample + wash water & g & 166.94 & 336.34 & 277.53 & 318.36 & 200.50 \\
\hline Weight of sample & g & 128.90 & 298.39 & 239.08 & 279.60 & 162.49 \\
\hline Approx. density of sample & $g / m$ & 1.10 & 1.10 & $\div 1.10$ & 1.10 & 1.10 \\
\hline Volume of sample & $\mathrm{mI}$ & 117.2 & 271.3 & 217.3 & 254.2 & 147.7 \\
\hline Conc of $\mathrm{Hg}$ & mgliter (ppm) & 405.0 & 1.6 & 1.7 & 2.6 & 2.5 \\
\hline $\mathrm{Hg}$ collected & $\mathrm{mg}$ & 47.5 & 0.4 & 0.4 & 0.7 & 0.4 \\
\hline
\end{tabular}

Total $\mathrm{Hg}$ collected $\square \mathrm{mg} \quad 49.3$

DRY GAS SAMPLE: Gas Volume $\quad$ cuft 47.60

MERCURY CONCENTRATION MEASURED AT FAVC INLET:

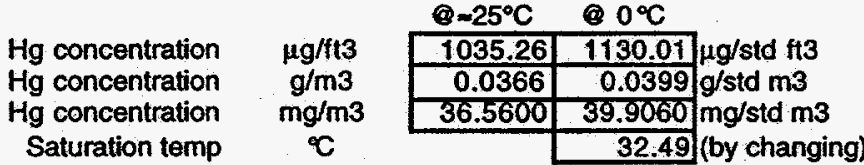

MERCURY CONCENTRATION AT SRATC EXIT CALCULATED FROM MEASURED FAVC INLET:

\begin{tabular}{|c|c|c|c|}
\hline & & (2) $\approx 25^{\circ} \mathrm{C}$ & $0^{\circ} \mathrm{C}$ \\
\hline & & 1238.35 & $1351.69 \mu$ \\
\hline $\mathrm{Hg}$ & & $\begin{array}{r}0.0437 \\
43.7320\end{array}$ & $\begin{array}{r}0.0477 \\
47.7345\end{array}$ \\
\hline
\end{tabular}

MERCURY CONCENTRATION AT SATURATION (PROCESS BASIS, SRATC EXIT) :

Average SRATC Exit Temperature $\quad{ }^{\circ} \mathrm{C} 223.40$

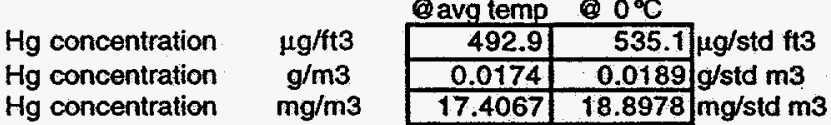

MERCURY CONCENTRATION AT FAVC INLET CALCULATED FROM SATURATION:

SRAT/FAVC Hlow ratio

FAVC Inlet Temperature

$\varnothing \quad 0.836$

Hg concentration

$\mathrm{Hg}$ concentration

$\mu \mathrm{g} / \mathrm{ft3}$

$\mathrm{g} / \mathrm{m} 3$

$\mathrm{Hg}$ concentration

$\mathrm{mg} / \mathrm{m} 3$

\begin{tabular}{|c|c|}
\hline QFAVC In T & (i) $0^{\circ} \mathrm{C}$ \\
\hline 416.1 & $447.4 \mu \mathrm{g} / \mathrm{std} \mathrm{ft} 3$ \\
\hline $\begin{array}{r}0.0147 \\
14.6957\end{array}$ & $\begin{array}{r}0.0158 \\
15.7986\end{array} \mathrm{~g} / \mathrm{std} \mathrm{m} / \mathrm{std} \mathrm{m3}$ \\
\hline
\end{tabular}

MEASURED / EXPECTED SATURATION

\subsection{6}




\section{APPENDIX}

IDMS RUN: $\frac{\text { PUREX7 }}{\text { COMMENTS PX7 9129-9133 Inlet }}$
OATE: $12 / 10-11 / 1994$
TIME: $15: 30-16: 45,03: 45-06: 45,09: 30-10: 30$

WASHED SAMPLE:

\begin{tabular}{|c|c|c|c|c|c|c|}
\hline & 9129 & \multicolumn{4}{|c|}{ IOMS \# ID-RUNHMPI- } \\
\hline & & $\mathrm{NaOH}$ & $\mathrm{NaOH}$ & KMnO4 & KMnO4 & KMnO4 \\
\hline Empty bottle & g & 38.79 & 38.64 & 38.69 & 38.35 & 37.91 \\
\hline Sample + wash water & g. & 245.38 & 245.64 & 321.39 & 329.64 & 136.63 \\
\hline Weight of sample & g] & 206.59 & 207.00 & 282.70 & 291.29 & 98.72 \\
\hline Approx density of sample & $g / m \mid$ & 1.10 & 1.10 & 1.10 & 1.10 & 1.10 \\
\hline Volume of sample & $\mathrm{m}$ & $\mid 87.8$ & 188.2 & 257.0 & 264.8 & 89.7 \\
\hline Conc of $\mathrm{Hg}$ & (ppm) & 47.4 & 35.8 & 0.1 & 10.3 & 1.6 \\
\hline
\end{tabular}

\begin{tabular}{r|r|r|r|r|r|r|} 
Hg collected & $\mathrm{mg}$ & 8.9 & 6.7 & 0.0 & 2.7 & 0.1 \\
\hline & $\%$ & 48.00 & 36.33 & 0.18 & 14.71 & 0.78 \\
\hline
\end{tabular}

Total $\mathrm{Hg}$ collected $\quad \mathbf{m g} \quad \mathbf{1 8 . 5}$

DRY GAS SAMPLE: Gas Volume $\quad$ cuttritr 16.70

\section{MERCURY CONCENTRATION MEASURED. AT FAVC INLET:}

\begin{tabular}{|c|c|c|c|c|}
\hline \multirow{4}{*}{$\begin{array}{l}\mathrm{Hg} \text { concentration } \\
\mathrm{Hg} \text { concentration } \\
\mathrm{Hg} \text { concentration } \\
\text { Saturation temo }\end{array}$} & & $Q=25^{\circ} \mathrm{C}$ & (e. $0^{\circ} \mathrm{C}$ & \multirow{4}{*}{$\begin{array}{l}\mu \mathrm{g} / \mathrm{std} \mathrm{ft} 3 \\
\mathrm{~g} / \mathrm{std} \mathrm{m3} \\
\mathrm{mg} / \mathrm{std} \mathrm{m3} \\
\text { (by changing) }\end{array}$} \\
\hline & & 1110.46 & 1212.09 & \\
\hline & & 0.0392 & 0.0428 & \\
\hline & $\mathrm{mg} / \mathrm{m} 3$ & 39.2154 & $\frac{42.8044}{33.37}$ & \\
\hline
\end{tabular}

MERCURY CONCENTRATION AT SRATC EXIT CALCULATED FROM MEASURED FAVC INLET:

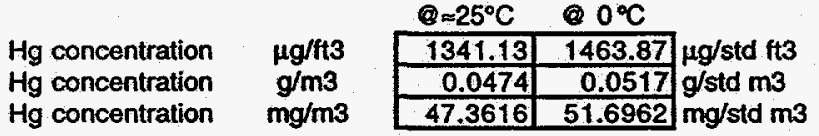

MERCURY CONCENTRATION AT SATURATION (PROCESS BASIS, SRATC EXIT) :

Average SRATC Exit Temperature

Hg concentration
Hg concentration
Hg concentration
C

25.00

Qavg temp $00^{\circ} \mathrm{C}$

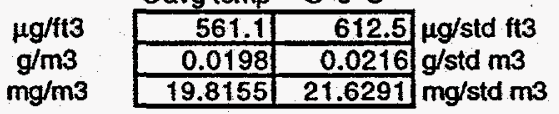

MERCURY CONCENTRATION AT FAVC INLET

SRAT/FAVC flow ratio

FAVC Inlet Temperature

$\mathrm{Hg}$ concentration

$\mathrm{Hg}$ concentration

$\mathrm{Hg}$ concentration

${ }^{\circ} \mathrm{C}$
$\mu \mathrm{g} / \mathrm{ft} 3$
$\mathrm{~g} / \mathrm{m} 3$
$\mathrm{mg} / \mathrm{m} 3$$$
\text { . }
$$$$
\begin{array}{|r|}
\hline 0.828 \\
\hline 21.50 \\
\hline
\end{array}
$$

QFAVCInT $0^{\circ} \mathrm{C}$

\begin{tabular}{|r|r|r|}
\hline 470.1 & 507.7 & $\mu \mathrm{g} / \mathrm{std} \mathrm{ft} 3$ \\
\hline 0.0166 & 0.0179 & $\mathrm{~g} / \mathrm{std} \mathrm{m3}$ \\
\hline 16.6021 & 17.9089 & $\mathrm{mg} / \mathrm{std} \mathrm{m3}$ \\
\hline
\end{tabular}

MEASURED / EXPECTED SATURATION 


\section{APPENDIX}

IDMS RUN: PUREX 7

COMMENTS PX7 9134-9138 Inlet

DATE: $12 / 1194$

TIME: 11:35-16:20

WASHED SAMPLE:

\begin{tabular}{|c|c|c|c|c|c|c|}
\hline & \multicolumn{2}{|c|}{ DMS \& DD-RUNAMPI- } & \multirow{2}{*}{9136} & \multirow{2}{*}{9137} & \\
\hline & & 9134 & 9135 & & & 9138 \\
\hline & & $\mathrm{NaOH}$ & $\mathrm{NaOH}$ & $\mathrm{KMnO4}$ & $\mathrm{KMnO} 4$ & $\mathrm{KMnO4}$ \\
\hline Empty bottie & $\mathrm{g}$ & 38.36 & 38.45 & 38.76 & 38.60 & 38.07 \\
\hline Sample + wash water & g) & 247.03 & 290.51 & 413.11 & 397.67 & 138.99 \\
\hline Weight of sample & g] & 208.67 & 252.06 & 374.35 & 359.07 & 100.92 \\
\hline Approx. density of sample & $g / m !$ & 1.10 & 1.10 & 1.10 & 1.10 & 1.10 \\
\hline Volume of sample & $\mathrm{m}$ & 189.7 & 229.1 & 340.3 & 326.4 & 91.7 \\
\hline
\end{tabular}

\begin{tabular}{lllllllll} 
Conc of $\mathrm{Hg}$ & $\mathbf{m g}$ miter $(\mathrm{ppm})$ & 134.0 & 1.7 & 5.1 & 5.2 & 0.2 \\
\hline
\end{tabular}

\begin{tabular}{rr|r|r|r|r|r|} 
Hg collected & $\mathrm{mg}$ & 25.4 & 0.4 & 1.7 & 1.7 & 0.0 \\
\cline { 2 - 7 }$\%$ of total collected & $\%$ & 86.89 & 1.30 & 5.91 & 5.84 & 0.07 \\
\cline { 2 - 7 }
\end{tabular}

Total $\mathrm{Hg}$ collected $\square \mathrm{mg} \quad 29.3$

DRY GAS SAMPLE: Gas Volume 16

MERCURY CONCENTRATION MEASURED AT FAVC INLET:

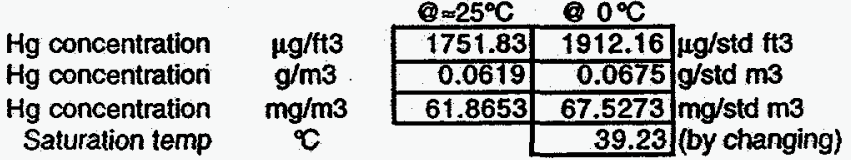

MERCURY CONCENTRATION AT SRATC EXIT CALCULATED FROM MEASURED FAVC INLET:

\begin{tabular}{|c|c|c|c|}
\hline & & $Q=25^{\circ} \mathrm{C}$ & (Q) $0^{\circ} \mathrm{C}$ \\
\hline concentra & $\mu g / t$ & 2131.18 & $2326.23 \mu \mathrm{g} / \mathrm{std} \mathrm{ft} 3$ \\
\hline $\begin{array}{l}\mathrm{Hg} \text { concentration } \\
\mathrm{Hg} \text { concentration }\end{array}$ & $\begin{array}{c}\mathrm{g} / \mathrm{m} 3 \\
\mathrm{mg} / \mathrm{m3}\end{array}$ & $\begin{array}{r}0.0753 \\
75.2619\end{array}$ & \begin{tabular}{r|r|r|r|}
0.0822 & $\mathrm{~g} / \mathrm{std} 3$ \\
82.1500 & $\mathrm{mg} / \mathrm{std} \mathrm{m3}$
\end{tabular} \\
\hline
\end{tabular}

MERCURY CONCENTRATION AT SATURATION (PROCESS BASIS, SRATC EXIT) :

Average SRATC Exit Temperature

Hg concentration

$\mathrm{Hg}$ concentration

$\mathrm{Hg}$ concentration

$\subset \quad 23.40$

$\underset{\mathrm{g} / \mathrm{m} 3}{\mathrm{~m} / \mathrm{ft} 3}$

$\mathrm{mg} / \mathrm{m} 3$

MERCURY CONCENTRATION AT FAVC INLET CALCULATED FROM SATURATION:

SRAT/FAVC flow ratio

FAVC intet Temperature

Hg concentration

Hg concentration

$\mathrm{Hg}$ concentration

$\mu \mathrm{g} / \mathrm{ft} 3$

$g / m 3$

$\mathrm{mg} / \mathrm{m3}$
${ }^{\circ}$

BFAVC InT $00^{\circ} \mathrm{C}$

$0.0155 \mathrm{~g} / \mathrm{std} \mathrm{m} 3$

\begin{tabular}{lll}
\hline 14.4743 & 15.5340 & $\mathrm{mg} / \mathrm{std} \mathrm{m} 3$
\end{tabular}

MEASURED / EXPECTED S SATURATION 


\section{APPENDIX}

IDMS RUN: PUREX7 COMMENTS PX7 9139-9143 Inlet

DATE: $12 / 11 / 94$

TIME: 16:45-21:45

WASHED SAMPLE:

\begin{tabular}{|c|c|c|c|c|c|c|}
\hline \multirow{3}{*}{\multicolumn{2}{|c|}{ E: }} & \multicolumn{5}{|c|}{ IDMS \# ID-RUNAMPI- } \\
\hline & & 9139 & 9140 & 9141 & 9142 & 9143 \\
\hline & & $\mathrm{NaOH}$ & $\mathrm{NaOH}$ & KMnO4 & KMnO4 & KMnO4 \\
\hline Empty bottle & g & 37.90 & 38.70 & 38.90 & 38.30 & 37.90 \\
\hline Sample + wash water & $g$ & 285.90 & 297.60 & 309.40 & 315.40 & 322.10 \\
\hline
\end{tabular}

\begin{tabular}{r|r|r|r|r|r|r|} 
Weight of sample & $g$ & 248.00 & 258.90 & 270.50 & 277.10 & 284.20 \\
\cline { 2 - 6 } & Approx. density of sample \\
Volume of sample & $\mathrm{g} / \mathrm{m} /$ & 1.10 & 1.10 & 1.10 & 1.10 & 1.10 \\
\hline $\mathrm{m} /$ & 225.5 & 235.4 & 245.9 & 251.9 & 258.4 \\
\hline
\end{tabular}

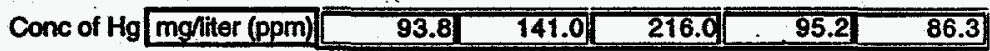

\begin{tabular}{|c|c|c|c|c|c|c|}
\hline $\begin{array}{l}\mathrm{Hg} \text { collected } \\
\% \text { of total collected }\end{array}$ & $\frac{\mathrm{mg}}{\%}$ & \begin{tabular}{r|}
21.1 \\
13.76
\end{tabular} & \begin{tabular}{r|}
33.2 \\
2159
\end{tabular} & \begin{tabular}{r|}
53.1 \\
34.55 \\
\end{tabular} & $\frac{24.0}{15.60}$ & $\frac{22.3}{14.50}$ \\
\hline
\end{tabular}

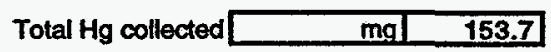

DRY GAS SAMPLE: Gas Volume $\quad$ cuff 54.10

MERCURY CONCENTRATION MEASURED AT FAVC INLET:

$\mathrm{Hg}$ concentration

Hg concentration

$\mathrm{Hg}$ concentration

Saturation temp

$\mu g / t$ t3

$g / m 3$

$m g / m 3$

Q $25^{\circ} \mathrm{C}$ ( $0^{\circ} \mathrm{C}$

\begin{tabular}{|c|c|c|}
\hline 2841.57 & 3101.63 & $\mu g$
\end{tabular}$/ \mathrm{std} \mathrm{ft3}$

\begin{tabular}{|l|l|l|l}
\hline 100.3490 & 0.1095 & $\mathrm{~g} / \mathrm{std} \mathrm{m} 3$ \\
\hline
\end{tabular}

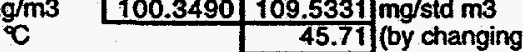

MERCURY CONCENTRATION AT SRATC EXIT CALCULATED FROM MEASURED FAVC INLET:

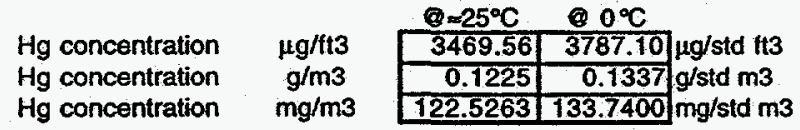

MERCURY CONCENTRATION AT SATURATION (PROCESS BASIS, SRATC EXIT) :

Average SRATC Exit Temperature

C

19.50

\begin{tabular}{|c|c|c|c|}
\hline & & Q avg temp & (2) $0^{\circ} \mathrm{C}$ \\
\hline & & 357.2 & $382.6 \mu$ \\
\hline & & 0.0126 & $0.0135 \mathrm{~g} / \mathrm{std} \mathrm{r}$ \\
\hline & & 12.6127 & $13.5131 \mathrm{mg}$ \\
\hline
\end{tabular}

MERCURY CONCENTRATION AT FAVC INLET CALCULATED FROM SATURATION:

SRAT/FAVC flow ratio

FAVC Inlet Temperature

C $\quad 0.819$

19.20

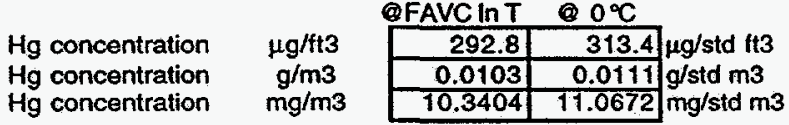

MEASURED / EXPECTED SATURATION

9.897 


\section{APPENDIX}

IDMS RUN: PUREX7

COMMENTS PX7 9144-9148 Inlet

DATE: $12 / 11-12 / 1994$

TIME: 23:00-04:00

WASHED SAMPLE:

IDMS \# ID-RUN-IMPI

\begin{tabular}{|c|c|c|c|c|c|c|}
\hline & \\
\hline & & 9144 & 9145 & 9146 & 9147 & 9148 \\
\hline & & $\mathrm{NaOH}$ & $\mathrm{NaOH}$ & $\mathrm{KMnO4}$ & $\mathrm{KMnO4}$ & KMnO4 \\
\hline Empty bottle & $\mathrm{g}$ & 38.39 & 37.98 & 38.54 & 38.29 & 38.21 \\
\hline Sample + wash water & $g$ & 236.08 & 257.60 & 282.96 & 278.02 & 282.00 \\
\hline
\end{tabular}

Weight of sample

\begin{tabular}{|r|r|r|r|r|r|}
\hline$g$ & 197.69 & 219.62 & 244.42 & 239.73 & 243.79 \\
\hline $\mathrm{g} / \mathrm{m}$ & 1.10 & 1.10 & 1.10 & 1.10 & 1.10 \\
\hline $\mathrm{m} /$ & 179.7 & 199.7 & 222.2 & 217.9 & 221.6 \\
\hline
\end{tabular}

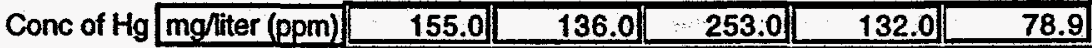

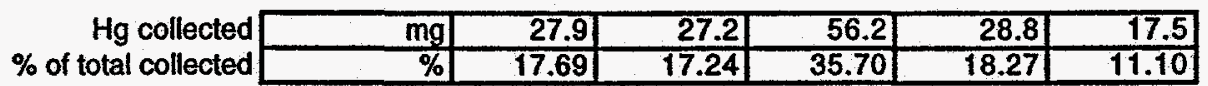

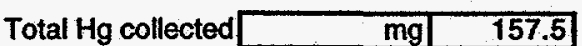

DRY GAS SAMPLE: Gas Volume $\quad$ cu tt $\quad 42.70$

MERCURY CONCENTRATION MEASURED AT FAVC INLET:

Hg concentration

Hg concentration

Hg concentration

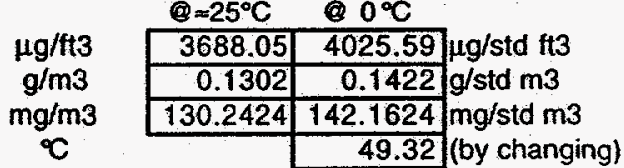

MERCURY CONCENTRATION AT SRATC

EXIT CALCULATED FROM MEASURED FAVC INLET:

$\mathrm{Hg}$ concentration

$\mathrm{Hg}$ concentration

$\mu \mathrm{g} / \mathrm{tt} 3$

$\mathrm{g} / \mathrm{m} 3$

$\mathrm{Hg}$ concentration

$\mathrm{mg} / \mathrm{m} 3$

(2) $=25^{\circ} \mathrm{C}$

(2) $0^{\circ} \mathrm{C}$

$\begin{array}{lll}4519.67 & 4933.32 & \mu \mathrm{g} / \mathrm{std} \mathrm{ft} 3\end{array}$

\begin{tabular}{r|r}
\hline 0.1596 & 0.1742 \\
\hline
\end{tabular}$/ \mathrm{std} \mathrm{m3}$

\begin{tabular}{|l|l|l|}
\hline 159.6108 & 174.2186 & $\mathrm{mg} / \mathrm{std} \mathrm{m} 3$ \\
\hline
\end{tabular}

MERCURY CONCENTRATION AT SATURATION (PROCESS BASIS, SRATC EXIT) :

Average SRATC Exit Temperature

${ }^{\circ} \mathrm{C}$

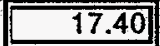

$\mathrm{Hg}$ concentration

$\mathrm{Hg}$ concentration

$\underset{\mathrm{mg} / \mathrm{m} 3}{\mu \mathrm{m} 3}$

Gavg temp @ $0^{\circ} \mathrm{C}$

$\mathrm{Hg}$ concentration

\begin{tabular}{|r|r|r|}
\hline 299.1 & 318.2 & $\mu g / s t d ~ f t 3$ \\
\hline 0.0106 & 0.0112 & $\mathrm{~g} / \mathrm{std} \mathrm{m} 3$ \\
\hline 10.5643 & 11.2372 & $\mathrm{mg} / \mathrm{std} \mathrm{m3}$
\end{tabular}

MERCURY CONCENTRATION AT FAVC INLET CALCULATED FROM SATURATION:

SRAT/FAVC flow ratio

FAVC Inlet Temperature

$\mathrm{Hg}$ concentration

$\mathrm{Hg}$ concentration

$\mathrm{Hg}$ concentration
${ }^{\circ}$

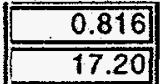

@FAVC InT @ $0^{\circ} \mathrm{C}$

$\mu \mathrm{g} / \mathrm{ft} 3$

$\mathrm{g} / \mathrm{m} 3$

$\mathrm{mg} / \mathrm{m} 3$
$244.3 \quad 259.7 \mu \mathrm{g} / \mathrm{std} \mathrm{H} 3$

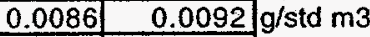

$\begin{array}{lll}8.6264 & 9.1696 & \mathrm{mg} / \mathrm{std} \mathrm{m} 3\end{array}$

MEASURED / EXPECTED @ SATURATION

15.504 


\section{APPENDIX}

IDMS RUN: PUREX 7

COMMENTS PX7 9149-9153 Inlet

DATE: $12 / 12 / 94$

TIME: 04:15-09:45

WASHED SAMPLE:

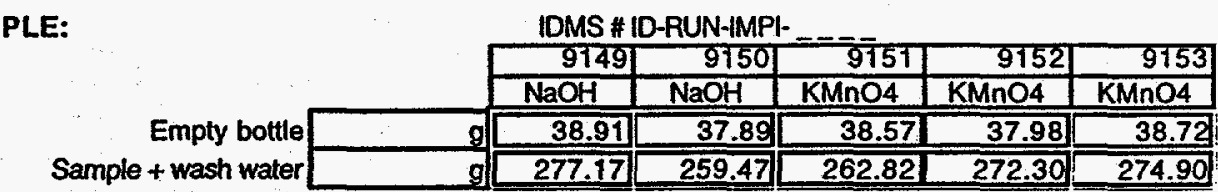

\begin{tabular}{|c|c|c|c|c|c|c|}
\hline Weight of sample & g] & 238.26 & 221.58 & 224.25 & 234.32 & 236.18 \\
\hline Approx. density of sample & $g / m$ & 1.10 & 1.10 & 1.10 & 1.10 & 1.10 \\
\hline Volume of sample & mol & 216.6 & 201.4 & 203.9 & 213.0 & 214.7 \\
\hline
\end{tabular}

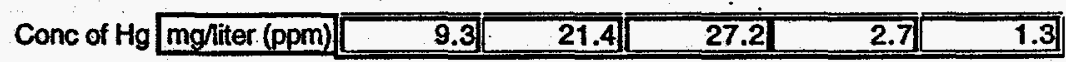

\begin{tabular}{r|r|r|r|r|r|r|} 
Hg collected & $\mathrm{mg}$ & 2.0 & 4.3 & 5.5 & 0.6 & 0.3 \\
\hline$\%$ of total collected & 15.85 & 33.85 & 43.54 & 4.52 & 2.24 \\
\hline
\end{tabular}

Total Hg collected $\square \quad \mathrm{mg} \quad 12.7$

DRY GAS SAMPLE: Gas Volume

MERCURY CONCENTRATION MEASURED AT FAVC INLET:

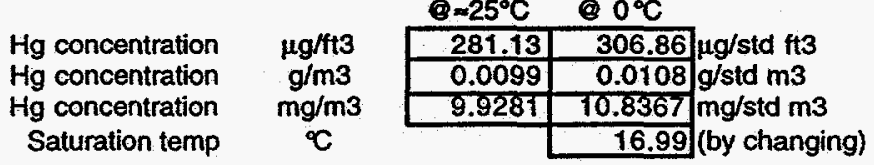

MERCURY CONCENTRATION AT SRATC EXIT CALCULATED FROM MEASURED FAVC INLET:

\begin{tabular}{|c|c|c|c|}
\hline & & $Q=25^{\circ} \mathrm{C}$ & (9) $0^{\circ} \mathrm{C}$ \\
\hline $\mathrm{Hg}$ concentration & $\mu \mathrm{g} / \mathrm{f}$ & 344.10 & $375.59 \mu \mathrm{g} / \mathrm{std} \mathrm{ft} 3$ \\
\hline $\begin{array}{l}\mathrm{Hg} \text { concentration } \\
\mathrm{Hg} \text { concentration }\end{array}$ & $\begin{array}{c}\mathrm{g} / \mathrm{m} 3 \\
\mathrm{mg} / \mathrm{m3}\end{array}$ & $\begin{array}{r}0.0122 \\
12.1519\end{array}$ & $\frac{0.0133}{13.2640} \mathrm{~g} / \mathrm{std} \mathrm{m} \mathrm{m} / \mathrm{std} \mathrm{m3}$ \\
\hline
\end{tabular}

MERCURY CONCENTRATION AT SATURATION (PROCESS BASIS, SRATC EXIT) : Average SRATC Exit Temperature $C \mathrm{C}$

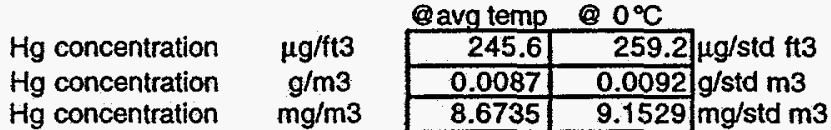

MERCURY CONCENTRATION AT FAVC INLET CALCULATED FROM SATURATION:

SRAT/FAVC How ratio

FAVC Inlet Temperature

C 15.817

Hg concentration

$\mathrm{Hg}$ concentration

$\mu \mathrm{g} / \mathrm{tt} 3$

$\mathrm{g} / \mathrm{m} 3$

$\mathrm{mg} / \mathrm{m} 3$

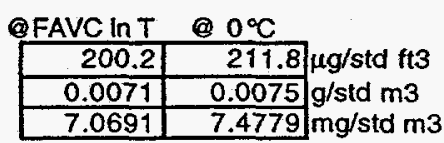

MEASURED / EXPECTED SATURATION

1.449 


\section{APPENDIX}

IDMS RUN: PUREX7

COMMENTS PX7 9154-9158 inlet

DATE: $12 / 13 / 94$

TIME: 03:30-08:30

WASHED SAMPLE:

IDMS \# ID-RUNAMPI-

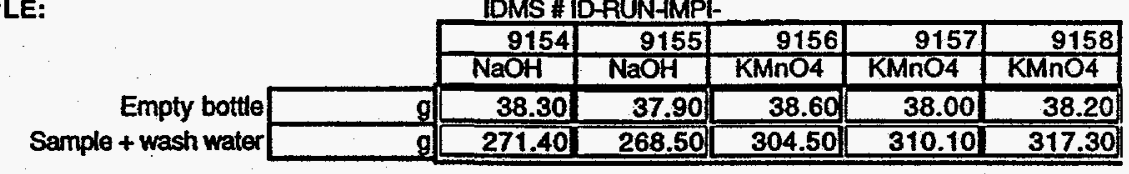

\begin{tabular}{r|r|r|r|r|r|r|}
\begin{tabular}{r} 
Weight of sample \\
\cline { 2 - 6 } Approx. density of sample
\end{tabular} & $\mathrm{g}$ & 233.10 & 230.60 & 265.90 & 272.10 & 279.10 \\
\cline { 2 - 7 } & $\mathrm{g} / \mathrm{m}$ & 1.10 & 1.10 & 1.10 & 1.10 & 1.10 \\
\hline $\mathrm{m}$ & 211.9 & 209.6 & 241.7 & 247.4 & 253.7 \\
\hline
\end{tabular}

Conc of $\mathrm{Hg}\left[\begin{array}{lllllll}\mathrm{mg} / \mathrm{iter}(\mathrm{ppm}) & 0.0 & 0.0 & 0.1 & 0.0 & 0.1 \\ \hline\end{array}\right.$

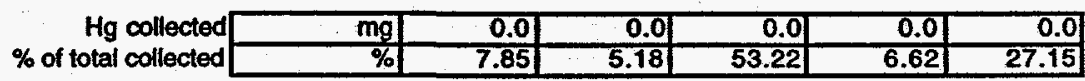

Total $\mathrm{Hg}$ collected $\quad \mathrm{mg} \quad 0.0$

DRY GAS SAMPLE: Gas Volume $\quad$ CUf: 31.20

MERCURY CONCENTRATION MEASURED AT FAVC INLET:

\begin{tabular}{|c|c|c|c|}
\hline \multirow{4}{*}{$\begin{array}{l}\mathrm{Hg} \text { concentration } \\
\mathrm{Hg} \text { concentration } \\
\mathrm{Hg} \text { concentration } \\
\text { Saturation temp }\end{array}$} & \multirow{4}{*}{$\begin{array}{c}\mu \mathrm{g} / \mathrm{ft} 3 \\
\mathrm{~g} / \mathrm{m3} \\
\mathrm{mg} / \mathrm{m3}\end{array}$} & \multicolumn{2}{|l|}{ (2) $\approx 25^{\circ} \mathrm{C}$} \\
\hline & & 1.56 & $1.70 \mathrm{\mu g} / \mathrm{std} \mathrm{ft} 3$ \\
\hline & & 0.0001 & $0.0001 \mathrm{~g} / \mathrm{std} \mathrm{m3}$ \\
\hline & & 0.0550 & $\frac{0.0600}{-31.51}$ (by changing) \\
\hline
\end{tabular}

MERCURY CONCENTRATION AT SRATC EXIT CALCULATED FROM MEASURED FAVC INLET:

\begin{tabular}{|c|c|c|c|}
\hline & & Q $=25^{\circ} \mathrm{C}$ & Q $0^{\circ} \mathrm{C}$ \\
\hline $\mathrm{Hg}$ concentration & $\mu \mathrm{g} / \mathrm{tt}$ & 1.97 & $2.15 \mu \mathrm{g} / \mathrm{std} \mathrm{ft3}$ \\
\hline $\mathrm{Hg}$ concentration & $\mathrm{g} / \mathrm{m} 3$ & 0.0001 & $0.0001 \mathrm{~g} / \mathrm{std} \mathrm{m3}$ \\
\hline g concentration & $\mathrm{mg} / \mathrm{m} 3$ & 0.0695 & $0.0758 \mathrm{mg} / \mathrm{std} \mathrm{m} 3$ \\
\hline
\end{tabular}

MERCURY CONCENTRATION AT SATURATION (PROCESS BASIS, SRATC EXIT) : Average SRATC Exit Temperature ${ }^{\circ} \mathrm{C}$

\begin{tabular}{|c|c|c|c|}
\hline & & Qavg temp & $00^{\circ} \mathrm{C}$ \\
\hline $\mathrm{Hg}$ concentration & & 363.2 & $389.4] \mu \mathrm{g} / \mathrm{std} \mathrm{ft} 3$ \\
\hline $\begin{array}{l}\mathrm{Hg} \text { concentration } \\
\mathrm{Hg} \text { concentration }\end{array}$ & $\begin{array}{c}\mathrm{g} / \mathrm{m} 3 \\
\mathrm{mg} / \mathrm{m} 3\end{array}$ & $\begin{array}{r}0.0128 \\
128256\end{array}$ & $\frac{0.0138}{13.7506} \mathrm{~g} / \mathrm{std} \mathrm{ma} / \mathrm{std} \mathrm{m}^{3}$ \\
\hline
\end{tabular}

MERCURY CONCENTRATION AT FAVC INLET CALCULATED FROM SATURATION:

SRAT/FAVC flow ratio

FAVC Inlet Temperature

${ }^{\circ}$

0.792

16.50

Hg concentration

$\mathrm{Hg}$ concentration

$\mathrm{Hg}$ concentration
$\mu \mathrm{g} / \mathrm{ft} 3$
$\mathrm{g} / \mathrm{m} 3$
$\mathrm{mg} / \mathrm{m} 3$

QFAVCInT $0^{\circ} \mathrm{C}$

\begin{tabular}{|r|r|l|}
\hline 290.8 & 308.4 & $\mathrm{\mu g} / \mathrm{std} \mathrm{ft} 3$ \\
\hline 0.0103 & 0.0109 & $\mathrm{~g} / \mathrm{std} \mathrm{m3}$ \\
\hline 10.2701 & 10.8904 & $\mathrm{mg} / \mathrm{std} \mathrm{m3}$ \\
\hline
\end{tabular}

MEASURED / EXPECTED SATURATION

0.006 
WSRC-TR-96-352, Rev. 0

\section{APPENDIX}

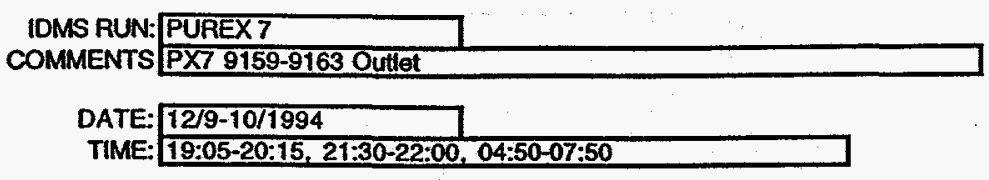

WASHED SAMPLE:

\begin{tabular}{|c|c|c|c|c|c|c|}
\hline \multirow{2}{*}{\multicolumn{2}{|c|}{$m$}} & & & & & \\
\hline & & $\frac{9159}{\mathrm{NaOH}}$ & $\frac{9160}{\mathrm{NaOH}}$ & $\frac{9161}{\mathrm{KMnO4}}$ & $\frac{9162}{\mathrm{KMnO4}}$ & $\begin{array}{c}9163 \\
\text { KMnO4 }\end{array}$ \\
\hline Emply bottie & g] & 38.95 & 38.58 & 37.96 & 37.98 & 38.53 \\
\hline Sample + wash water & g & 269.46 & 205.43 & 255.02 & 254.27 & 267.55 \\
\hline Weight of sample & g & 230.51 & 166.85 & 217.06 & 216.29 & 229.02 \\
\hline Approx. density of sample & $\mathrm{g} / \mathrm{ml}$ & 1.10 & 1.10 & 1.10 & 1.10 & 1.10 \\
\hline Volume of sample & mil & 209.6 & 151.7 & 197.3 & 196.6 & 208.2 \\
\hline Conc of $\mathrm{Hg}$ & mg/iter (ppm) & 0.3 & 0.0 & 0.0 & 0.0 & 0.0 \\
\hline $\mathrm{Hg}$ collected & $\mathrm{mg}$ & 0.1 & 0.0 & 0.0 & 0.0 & 0.0 \\
\hline$\%$ of total collected & $\%$ & 78.32 & 270 & 7.02 & 5.11 & 6.84 \\
\hline
\end{tabular}

Total Hg collected $\square \quad \mathrm{mg} / \quad 0.1$

DRY GAS SAMPLE: Gas Volume $\quad$ cuft $\quad 34.40$

MERCURY CONCENTRATION MEASURED AT FAVC EXIT:

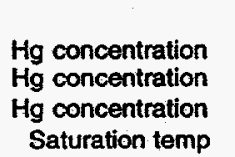

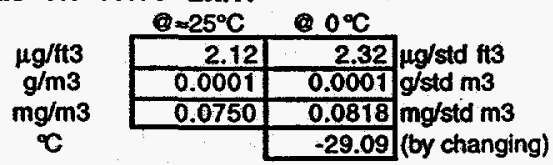

MERCURY CONCENTRATION AT SATURATION (PROCESS BASIS, FAVC EXIT) : Average FAVC Exit Temperature

${ }^{\circ} \quad 6.90$

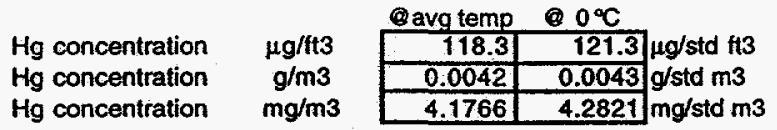

MEASURED / EXPECTED @ SATURATION 


\section{APPENDIX}

IDMS RUN: PUREX7

COMMENTS PX7 9164-9168 Outlet

DATE: $12 / 10 / 94$

TIME: 08:15-15:15

WASHED SAMPLE:

\begin{tabular}{|c|c|c|c|c|c|c|}
\hline \multirow[t]{3}{*}{$E:$} & & \multicolumn{2}{|c|}{ IDMS \# ID-RUN-IMPI } & & & \\
\hline & & 9164 & 9165 & 9166 & 9167 & 9168 \\
\hline & & $\mathrm{NaOH}$ & $\mathrm{NaOH}$ & $\mathrm{KMnO} 4$ & $\mathrm{KMnO4}$ & $\mathrm{KMnO4}$ \\
\hline Empty bottle & g & 38.15 & 38.32 & 38.62 & 38.12 & 37.88 \\
\hline Sample + wash water & $\mathrm{g}$ & 253.69 & 251.05 & 262.74 & 274.28 & 277.15 \\
\hline
\end{tabular}

\begin{tabular}{|r|r|r|r|r|r|r|} 
Weight of sample & $\mathrm{g}$ & 215.54 & 212.73 & 224.12 & 236.16 & 239.27 \\
\cline { 2 - 7 } Approx, density of sample & $\mathrm{g} / \mathrm{ml}$ & 1.10 & 1.10 & 1.10 & 1.10 & 1.10 \\
\hline Volume of sample & $\mathrm{m}$ & 195.9 & 193.4 & 203.7 & 214.7 & 217.5 \\
\hline
\end{tabular}

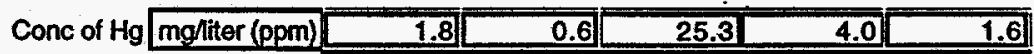

\begin{tabular}{r|r|r|r|r|r|r|}
$H g$ & $m g$ & 0.4 & 0.1 & 5.2 & 0.8 & 0.4 \\
\hline$\%$ of total collected & $\%$ & 5.28 & 1.65 & 75.47 & 12.42 & 5.19 \\
\hline
\end{tabular}

Total $\mathrm{Hg}$ collected $\square \mathrm{mgl} \quad 6.8$

DRY GAS SAMPLE: Gas Volume r cuftr $\quad 37.10$

MERCURY CONCENTRATION MEASURED AT FAVC EXIT:

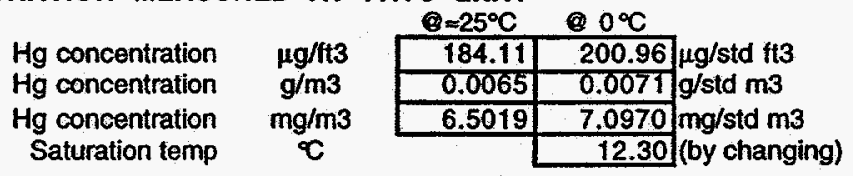

MERCURY CONCENTRATION AT SATURATION (PROCESS BASIS, FAVC EXIT) :

Average FAVC Exit Temperature

${ }^{\circ} \mathrm{C}$

$\begin{array}{cc}\mathrm{Hg} \text { concentration } & \mu \mathrm{g} / \mathrm{tt} 3 \\ \mathrm{Hg} \text { concentration } & \mathrm{g} / \mathrm{m} 3 \\ \mathrm{Hg} \text { concentration } & \mathrm{mg} / \mathrm{m} 3\end{array}$

MEASURED / EXPECTED @ SATURATION
7.20

Cavg temp $0^{\circ} \mathrm{C}$

\begin{tabular}{|r|r|r|}
\hline 121.6 & 124.8 & $\mathrm{gg} / \mathrm{std} \mathrm{ft} 3$ \\
\hline 0.0043 & 0.0044 & $\mathrm{~g} / \mathrm{std} \mathrm{m3}$ \\
\hline 4.2932 & 4.4063 & $\mathrm{mg} / \mathrm{std} \mathrm{m3}$ \\
\hline
\end{tabular}

USURED / EXPECTED Q SATURATION

1.611 


\section{APPENDIX}

IDMS RUN: PUREX 7
COMMENTS PX7 9169-9173 Outlet
DATE: $12 / 10-11 / 1994$
TIME: $15: 30-16: 45,03: 45-06: 45,09: 30-10: 30$

WASHED SAMPLE:

IDMS \# ID-RUN-MPP-

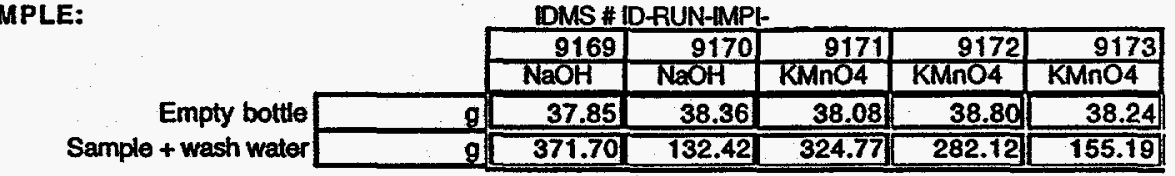

\begin{tabular}{|r|r|r|r|r|r|r|}
\begin{tabular}{r} 
Weight of sample \\
\cline { 2 - 6 } Approx. density of sample
\end{tabular} & $\mathrm{g}$ & 333.85 & 94.06 & 286.69 & 243.32 & 116.95 \\
\cline { 2 - 6 } & Volume of sample & 1.10 & 1.10 & 1.10 & 1.10 & 1.10 \\
\hline $\mathrm{m}$ & 303.5 & 85.5 & 260.6 & 221.2 & 106.3 \\
\hline
\end{tabular}

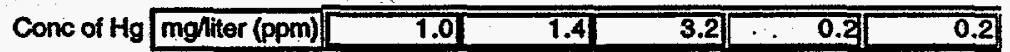

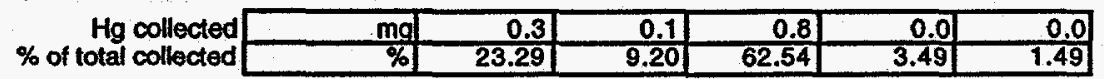

Total Hg collected $\quad$ mg 1.3

DRY GAS SAMPLE: Gas Volume $\quad$ cutt 30.20

MERCURY CONCENTRATION MEASURED AT FAVC EXIT:

$\begin{array}{cc}\text { Hg concentration } & \mu \mathrm{g} / \mathrm{ft} 3 \\ \mathrm{Hg} \text { concentration } & \mathrm{g} / \mathrm{m} 3 \\ \mathrm{Hg} \text { concentration } & \mathrm{mg} / \mathrm{m} 3 \\ \text { Saturation temp } & { }_{\mathrm{C}}\end{array}$

\begin{tabular}{|r|r|r|}
\hline \multicolumn{1}{c}{$05^{\circ} \mathrm{C}$} & $0 \% \mathrm{C}$ & \\
\hline 44.02 & 48.05 & $\mu \mathrm{g} / \mathrm{std} \mathrm{ft} 3$ \\
\hline 0.0016 & 0.0017 & $\mathrm{~g} / \mathrm{std} \mathrm{m3}$ \\
\hline 1.5546 & 1.6969 & $\mathrm{mg} / \mathrm{std} \mathrm{m3}$ \\
\hline & -2.47 & (by changing) \\
\hline
\end{tabular}

MERCURY CONCENTRATION AT SATURATION (PROCESS BASIS, FAVC EXIT) :

Average FAVC Exit Temperature

$\begin{array}{cc}\mathrm{Hg} \text { concentration } & \mu \mathrm{g} / \mathrm{ft} 3 \\ \mathrm{Hg} \text { concentration } & \mathrm{g} / \mathrm{m} 3 \\ \mathrm{Hg} \text { concentration } & \mathrm{mg} / \mathrm{m} 3\end{array}$

MEASURED / EXPECTED @ SATURATION
${ }^{\circ} \mathrm{7} \quad 7.10$

\begin{tabular}{|c|c|}
\hline avg temp & (20 $0^{\circ} \mathrm{C}$ \\
\hline 120.5 & $123.6 \mathrm{\mu g} / \mathrm{std} \mathrm{ft3}$ \\
\hline 0.0043 & $0.0044 \mathrm{~g} / \mathrm{std} \mathrm{m3}$ \\
\hline 4.2540 & $4.3646 \mathrm{mg} / \mathrm{std} \mathrm{m} 3$ \\
\hline
\end{tabular}

0.389 


\section{APPENDIX}

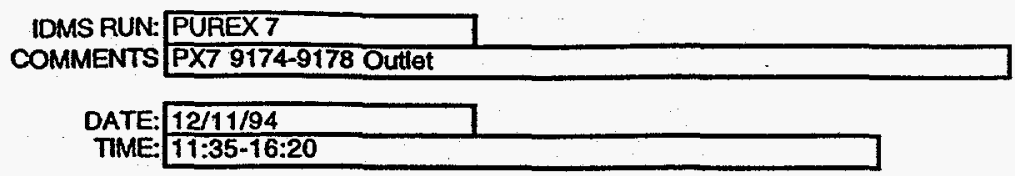

WASHED SAMPLE:

IDMS : IDRUN-IMPI-

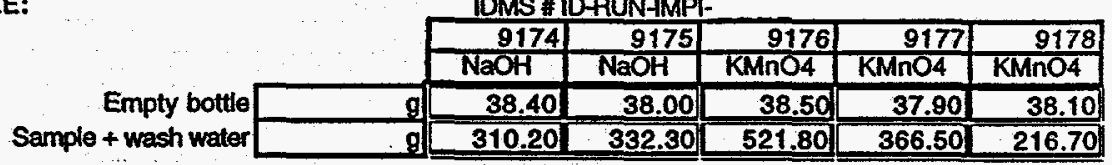

\begin{tabular}{|c|c|c|c|c|c|c|}
\hline Weight of sample & g & 271.80 & 294.30 & 483.301 & 328.60 & 178.60 \\
\hline Approx. density of sample & $\mathrm{g} / \mathrm{m}$ & 1.10 & 1.10 & 1.10 & 1.10 & 1.10 \\
\hline Volume of sample & $\mathbf{m}$ & 247.1 & 267.5 & 439.4 & 298.7 & 162.4 \\
\hline Conc of $\mathrm{Hg}$ & mgliter (ppm & 0.2 & 1.4 & 0.4 & 0.2 & 0.2 \\
\hline
\end{tabular}

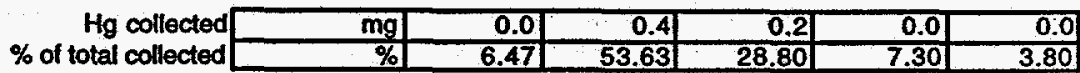

Total Hg collected

$\mathrm{mg} \quad 0.7$

DRY GAS SAMPLE:

Gas Volume

cu 1 15.20

MERCURY CONCENTRATION MEASURED AT FAVC EXIT:

$\begin{array}{cc}\mathrm{Hg} \text { concentration } & \mu \mathrm{g} / \mathrm{ft} 3 \\ \mathrm{Hg} \text { concentration } & \mathrm{g} / \mathrm{m} 3 \\ \mathrm{Hg} \text { concentration } & \mathrm{mg} / \mathrm{m} 3 \\ \text { Saturation temp } & { }_{\mathrm{C}}\end{array}$

\begin{tabular}{|c|c|}
\hline (e) $=25^{\circ} \mathrm{C}$ & (a $0^{\circ} \mathrm{C}$ \\
\hline 44.96 & $49.08 \mu \mathrm{g} / \mathrm{std} \mathrm{ft} 3$ \\
\hline 0.0016 & $0.0017 \mathrm{~g} / \mathrm{sid} \mathrm{m3}$ \\
\hline 1.5879 & $\frac{1.7333}{-2.26}$ (by changtd $\mathrm{m} 3$ \\
\hline
\end{tabular}

MERCURY CONCENTRATION AT SATURATION (PROCESS BASIS, FAVC EXIT) : Average FAVC Exit Temperature

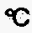
6.80

$\begin{array}{lc}\mathrm{Hg} \text { concentration } & \mu \mathrm{g} / \mathrm{ft} 3 \\ \mathrm{Hg} \text { concentration } & \mathrm{g} / \mathrm{m} 3 \\ \mathrm{Hg} \text { concentration } & \mathrm{mg} / \mathrm{m} 3\end{array}$

\begin{tabular}{|c|c|}
\hline Q avg temp & ( $0^{\circ} \mathrm{C}$ \\
\hline 117.2 & $120.1 \mu \mathrm{g} / \mathrm{std} \mathrm{ft} 3$ \\
\hline$\frac{0.0041}{4.1384}$ & $\frac{0.0042}{4.2414} \mathrm{~g} / \mathrm{std} \mathrm{m} 3 / \mathrm{std} \mathrm{m3}$ \\
\hline
\end{tabular}

MEASURED / EXPECTED SATURATION

0.409 


\section{APPENDIX}

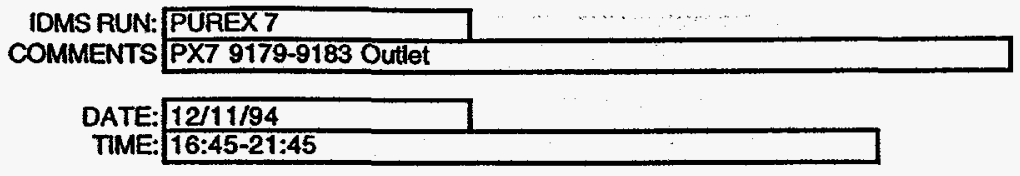

WASHED SAMPLE:

IDMS \# IDFUN-IMPI-

\begin{tabular}{|c|c|c|c|c|c|c|}
\hline & \multicolumn{2}{|c|}{ IDMS : ID-RUN-IMPI } & 0181 & $018 ?$ & 0183 \\
\hline & & $\mathrm{NaOH}$ & $\mathrm{NaOH}$ & KMnO4 & KMnO4 & KMnO4 \\
\hline Empty bottle & g & 38.20 & 37.80 & 38.80 & 38.50 & 38.60 \\
\hline Sample + wash water & g & 293.80 & 297.30 & 303.50 & 419.40 & 233.00 \\
\hline
\end{tabular}

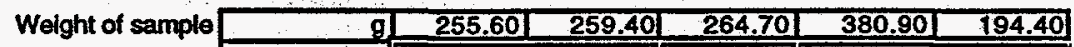

\begin{tabular}{|c|}
\hline Approx density of sample \\
\hline
\end{tabular}

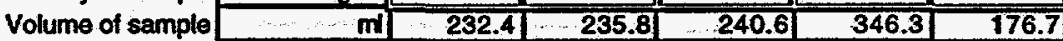

\begin{tabular}{rl|r|r|r|r|r|r|} 
Conc of $\mathrm{Hg}$ & $\mathrm{mg} / \mathrm{miter}(\mathrm{ppm})$ & 0.7 & 0.1 & 0.8 & 0.0 .4 & 1.3 \\
\hline
\end{tabular}

\begin{tabular}{r|r|r|r|r|r|r|} 
Hg collected & $\mathrm{mg}$ & 0.2 & 0.0 & 0.2 & 0.2 & 0.2 \\
\hline$\%$ & $\%$ & 20.67 & 2.66 & 27.12 & 20.37 & 29.18 \\
\hline
\end{tabular}

Total $\mathrm{Hg}$ collected $\square$

DRY GAS SAMPLE: Gas Volume $\quad$ CU At $\quad 66.90$

MERCURY CONCENTRATION MEASURED AT FAVC EXIT:

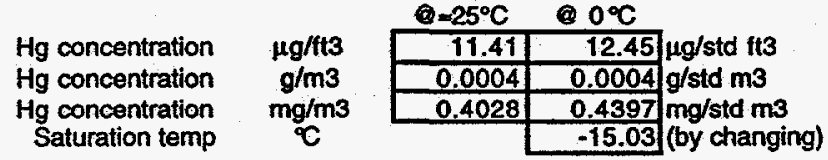

MERCURY CONCENTRATION AT SATURATION (PROCESS BASIS, FAVC EXIT) :

Average FAVC Exit Temperature

Hg concentration

$\mathrm{Hg}$ concentration

Hg concentration
C

6.00

$$
\begin{gathered}
\mu g / f t 3 \\
g / m 3 \\
m g / m 3
\end{gathered}
$$

Qavg temp $00^{\circ} \mathrm{C}$

\begin{tabular}{|r|r|r|}
\hline 108.9 & 111.2 & $\mu \mathrm{g} / \mathrm{std} \mathrm{ft} 3$ \\
\hline 0.0038 & 0.0039 & $\mathrm{~g} / \mathrm{std} \mathrm{m3}$ \\
\hline 3.8441 & 3.9286 & $\mathrm{mg} / \mathrm{std} \mathrm{m3}$ \\
\hline
\end{tabular}

MEASURED / EXPECTED SATURATION 0.112 
WSRC-TR-96-352, Rev. 0

\section{APPENDIX}

IDMS RUN: PUREX 7

COMMENTS PX7 9184-9188 Outlet

DATE: उयाTन12/2994

TIME: 23:00004:00

WASHED SAMPLE:

\begin{tabular}{|c|c|c|c|c|c|c|}
\hline \multirow{3}{*}{\multicolumn{2}{|c|}{ MPLE: }} & \multicolumn{5}{|c|}{ IDMS \# ID-RUN-MPI- } \\
\hline & & 9184 & 9185 & 9186 & 9187 & 9188 \\
\hline & & $\mathrm{NaOH}$ & $\mathrm{NaOH}$ & $\mathrm{KMnO} 4$ & $\mathrm{KMnO4}$ & $\mathrm{KMnO4}$ \\
\hline Empty bottle & g & 37.76 & 38.26 & 37.96 & 38.68 & 37.92 \\
\hline Sample + wash water & g. & 243.58 & 248.97 & 294.49 & 430.97 & 92.95 \\
\hline Weight of sample & g & 205.82 & 210.71 & 256.53 & 392.29 & 55.03 \\
\hline Approx. densily of sampla & $g / n:$ & 1.10 & 1.10 & 1.10 & 1.10 & 1.10 \\
\hline Volume of sample & $m$ & 187.1 & 191.6 & 233.2 & 356.6 & 50.0 \\
\hline
\end{tabular}

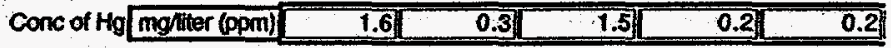

\begin{tabular}{rr|r|r|r|r|r|}
$H g$ & mg collected & 0.3 & 0.1 & 0.3 & 0.1 & 0.0 \\
\cline { 1 - 5 } & \% of total collected & 37.51 & 7.21 & 43.29 & 10.59 & 1.39 \\
\hline
\end{tabular}

Total $\mathrm{Hg}$ collected $\quad 0.8$

DRY GAS SAMPLE: Gas Volume $\quad$ cuft 52.80

MERCURY CONCENTRATION MEASURED AT FAVC EXIT:

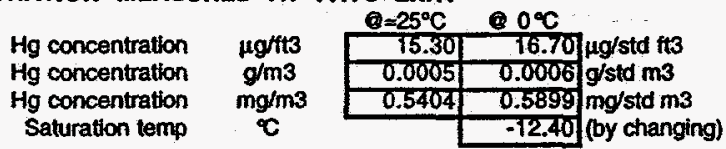

MERCURY CONCENTRATION AT SATURATION (PROCESS BASIS, FAVC EXIT) :

- Average FAVC Exit Temperature

Hg concentration

$\mathrm{Hg}$ concentration

$\mu g / f t 3$

$\mathrm{mg} / \mathrm{m} 3$

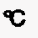

\begin{tabular}{|c|c|}
\hline Q avg temp & $90^{\circ} \mathrm{C}$ \\
\hline 90.3 & 91.7 $\mu \mathrm{g} / \mathrm{std} \mathrm{ft} 3$ \\
\hline 0.0032 & $0.0032 \mathrm{~g} / \mathrm{std} \mathrm{m3}$ \\
\hline 3.7906 & $3.2373 \mathrm{mg} / \mathrm{std} \mathrm{m3}$ \\
\hline
\end{tabular}

MEASURED / EXPECTED S SATURATION

0.182 
WSRC-TR-96-352, Rev. 0

\section{APPENDIX}

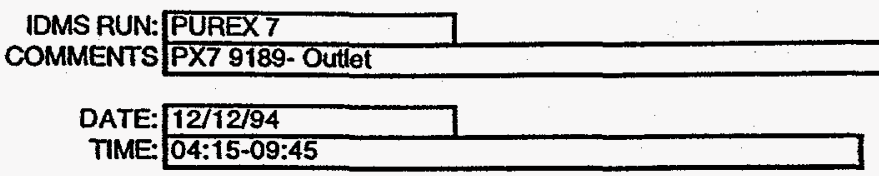

WASHED SAMPLE:

IDMS \# ID-RUN-IMPL

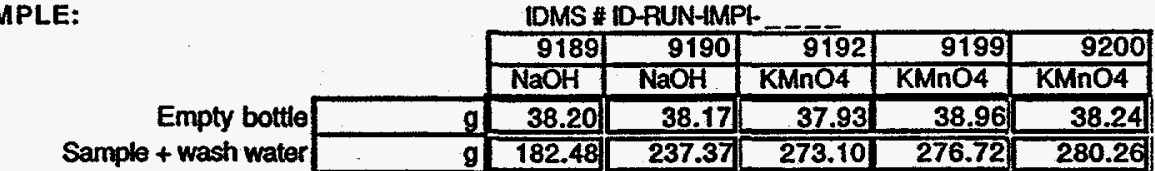

\begin{tabular}{|r|r|r|r|r|r|r|}
\hline \begin{tabular}{r} 
Weight of sample \\
\cline { 2 - 5 }
\end{tabular} & $\mathrm{g}$ & 144.28 & 199.20 & 235.17 & 237.76 & 242.02 \\
\hline Approx. density of sample & $g / \mathrm{m} \mid$ & 1.10 & 1.10 & 1.10 & 1.10 & 1.10 \\
\hline Volume of sample & $\mathrm{m}$ & 131.2 & 181.1 & 213.8 & 216.1 & 220.0 \\
\hline
\end{tabular}

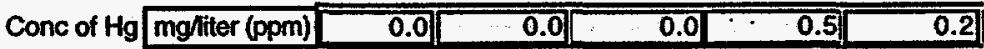

\begin{tabular}{r|r|r|r|r|r|r|} 
Hg collected & mg & 0.0 & 0.0 & 0.0 & 0.1 & 0.0 \\
\hline$\%$ of total collected & $\%$ & 0.85 & 0.47 & 3.19 & 72.93 & 22.56 \\
\hline
\end{tabular}

Total Hg collected $\begin{array}{r}\mathrm{mg} \\ \mathrm{O}\end{array}$

DRY GAS SAMPLE: Gas Volume cuft 54.90

MERCURY CONCENTRATION MEASURED AT FAVC EXIT:

\begin{tabular}{|c|c|c|c|}
\hline \multirow{4}{*}{$\begin{array}{l}\mathrm{Hg} \text { concentration } \\
\mathrm{Hg} \text { concentration } \\
\mathrm{Hg} \text { concentration } \\
\text { Saturation temp }\end{array}$} & \multirow{4}{*}{$\begin{array}{c}\mu \mathrm{g} / \mathrm{ft3} \\
\mathrm{g} / \mathrm{m} 3 \\
\mathrm{mg} / \mathrm{m} 3\end{array}$} & \multirow{2}{*}{$\frac{Q=25^{\circ} \mathrm{C}}{2.81}$} & $80^{\circ}$ \\
\hline & & & $3.06 \mu \mathrm{g} / \mathrm{std} \mathrm{ft} 3$ \\
\hline & & 0.0001 & $0.0001 \mathrm{~g} / \mathrm{std} \mathrm{m} 3$ \\
\hline & & 0.0991 & $\begin{array}{l}0.1082 \text { mg/std m3 } \\
-26.87 \text { (by changing) }\end{array}$ \\
\hline
\end{tabular}

MERCURY CONCENTRATION AT SATURATION (PROCESS BASIS, FAVC EXIT) : Average FAVC Exit Temperature

${ }^{\circ} \quad 3.00$

Hg concentration Qavatem Q $0^{\circ} \mathrm{C}$

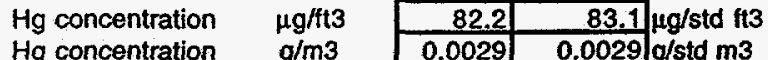

\begin{tabular}{llrl}
$\mathrm{Hg}$ concentration & $\mathrm{mg} / \mathrm{m3}$ & 2.9036 & 2.9355 \\
\cline { 2 - 3 } & &
\end{tabular}

MEASURED / EXPECTED @ SATURATION 


\section{APPENDIX}

IDMS RUN: PUREX 7

COMMENTS PX7 9201-9205 Outlet

DATE: $12 / 12 / 94$

TIME: 03:30-08:30

WASHED SAMPLE:

IDMS \# ID-RUN-IMPF-

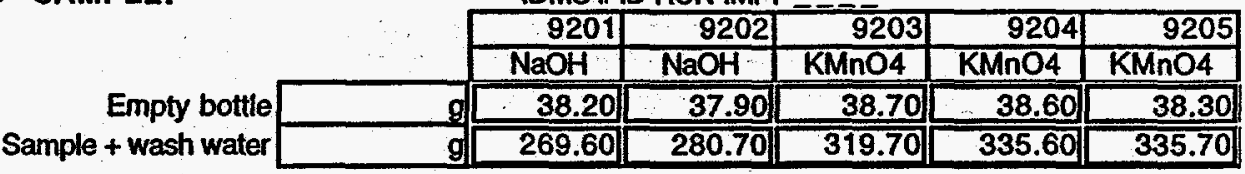

eight sample

\begin{tabular}{|r|r|r|r|r|r|}
\hline $\mathrm{g}$ & 231.40 & 242.80 & 281.00 & 297.00 & 297.40 \\
\hline $\mathrm{g} / \mathrm{m}$ & 1.10 & 1.10 & 1.10 & 1.10 & 1.10 \\
\hline $\mathrm{m}$ & 210.4 & 220.7 & 255.5 & 270.0 & 270.4 \\
\hline
\end{tabular}

Volume of sample

ml $=210.4 \quad 220.7$

255.5

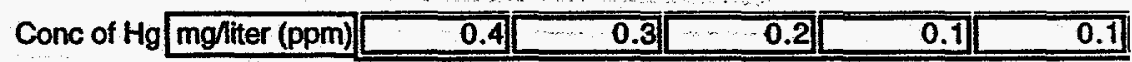

\begin{tabular}{rr|r|r|r|r|r|} 
Hg collected & $\mathrm{mg}$ & 0.1 & 0.1 & 0.0 & 0.0 & 0.0 \\
\cline { 2 - 7 } & $\%$ of total collected & 36.57 & 22.30 & 18.91 & 8.14 & 14.08 \\
\cline { 2 - 6 }
\end{tabular}

Total $\mathrm{Hg}$ collected $\square \mathrm{mg} / 0.3$

DRY GA

Gas Volume

cu ftr 50.00

MERCURY CONCENTRATION MEASURED AT FAVC EXIT:

\begin{tabular}{|c|c|c|c|c|}
\hline \multirow{4}{*}{$\begin{array}{l}\mathrm{Hg} \text { concentration } \\
\mathrm{Hg} \text { concentration } \\
\text { Hg concentration } \\
\text { Saturation temp }\end{array}$} & \multirow{4}{*}{$\begin{array}{c}\mu \mathrm{g} / \mathrm{ft} 3 \\
\mathrm{~g} / \mathrm{m} 3 \\
\mathrm{mg} / \mathrm{m} 3 \\
{ }^{\circ}\end{array}$} & $\mathrm{C}=25^{\circ} \mathrm{C}$ & (2) $0^{\circ} \mathrm{C}$ & \\
\hline & & 5.11 & 5.58 & $\mu \mathrm{g} / \mathrm{std} \mathrm{ft} 3$ \\
\hline & & 0.0002 & 0.0002 & $\mathrm{~g} / \mathrm{std} \mathrm{m} 3$ \\
\hline & & 0.1804 & $\frac{0.1969}{-21.95}$ & $\begin{array}{l}\text { mg/std m3 } \\
\text { (by changing) }\end{array}$ \\
\hline
\end{tabular}

MERCURY CONCENTRATION AT SATURATION (PROCESS BASIS, FAVC EXIT) : verage FAVC Exit Temperature

$$
{ }^{\circ} \mathrm{C} \quad 5.10
$$

$\mathrm{Hg}$ concentration

$\mathrm{Hg}$ concentration

$\mu \mathrm{g} / \mathrm{ft} 3$

$\mathrm{g} / \mathrm{m} 3$

Qavg temp $00^{\circ} \mathrm{C}$

$\mathrm{Hg}$ concentration $\mathrm{mg} / \mathrm{m} 3$

\begin{tabular}{|c|c|c|}
\hline Q avg temp & (8) $0^{\circ} \mathrm{C}$ & \\
\hline 100.1 & 102.0 & $\mu \mathrm{g} / \mathrm{std} \mathrm{ft} 3$ \\
\hline 0.0035 & 0.0036 & $\mathrm{~g} / \mathrm{std} \mathrm{m3}$ \\
\hline 3.5361 & 3.6022 & $\mathrm{mg} / \mathrm{std} \mathrm{m} 3$ \\
\hline
\end{tabular}

MEASURED / EXPECTED @ SATURATION

0.055 


\section{APPENDIX}

IDMS RUN: PUREX6

COMMENTS Set 1

DATE: $12 / 7 / 93$

TIME: 13:55 PM - 19:00 PM

WASHED SAMPLE:

IDMS \# ID-RUN-IMPI-

\begin{tabular}{|c|c|c|c|c|c|c|}
\hline & -7420 & 7421 & 7422 & 7423 & 7424 \\
\hline & & $\mathrm{NaOH}$ & $\mathrm{NaOH}$ & $\mathrm{KMnO} 4$ & $\mathrm{KMnO} 4$ & $\mathrm{KMnO} 4$ \\
\hline r & g & 38.76 & 39.13 & 38.10 & 38.24 & 39.05 \\
\hline wash w & & 413.28 & 498.34 & 178.25 & 112.01 & 88.95 \\
\hline
\end{tabular}

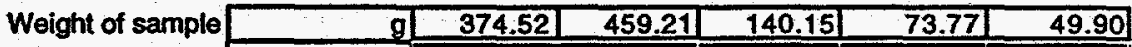

Approx. density of sample

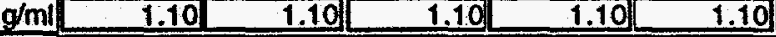

Volume of sample

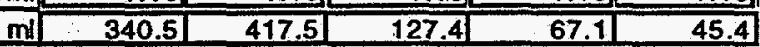

\begin{tabular}{lllllllllll} 
Conc of $\mathrm{Hg}$ & mg/iter (ppm) & 132.0 & 125.0 & 100.0 & 71.4 & 75.2 \\
\hline
\end{tabular}

\begin{tabular}{rr|r|r|r|r|r|} 
Hg collected & $\mathrm{mg}$ & 44.9 & 52.2 & 12.7 & 4.8 & 3.4 \\
\hline$\%$ of total collected & $\%$ & 38.07 & 44.20 & 10.79 & 4.06 & 2.89 \\
\hline
\end{tabular}

Total $\mathrm{Hg}$ collected

mg 118.1

DRY GAS SAMPLE: Gas Volume cu tt 132.50

MERCURY CONCENTRATION MEASURED AT FAVC EXIT:

\begin{tabular}{|c|c|c|c|c|}
\hline \multirow{4}{*}{$\begin{array}{l}\text { Hg concentration } \\
\text { Hg concentration } \\
\text { Hg concentration }\end{array}$} & & $\mathrm{Q} \approx 25^{\circ} \mathrm{C}$ & ब $0^{\circ} \mathrm{C}$ & \multirow{4}{*}{$\begin{array}{l}\mu g / s t d \text { ft3 } \\
g / s t d \text { m3 } \\
\text { mg/std m3 } \\
\text { (by changing) }\end{array}$} \\
\hline & $\mu g / t t 3$ & 891.06 & 972.62 & \\
\hline & & $\frac{0.0375}{31.4676}$ & $\frac{0.0345}{34.3476}$ & \\
\hline & & & & \\
\hline
\end{tabular}

MERCURY CONCENTRATION AT SATURATION (PROCESS BASIS, FAVC EXIT) :

\begin{tabular}{|c|c|c|c|c|}
\hline & & Q avg temp & $0^{\circ} \mathrm{C}$ & \\
\hline & $\mu g / f t$ & 214.6 & 225.3 & $\mu g / s t d \mathrm{ft3}$ \\
\hline oncentration & $\mathrm{g} / \mathrm{m} 3$ & 0.0076 & 0.0080 & $\mathrm{~g} / \mathrm{std} \mathrm{m} 3$ \\
\hline $\mathrm{Hg}$ concentration & $\mathrm{mg} / \mathrm{m} 3$ & 7.5799 & 7.9559 & $\mathrm{mg} / \mathrm{std} \mathrm{m}$ \\
\hline
\end{tabular}

MEASURED / EXPECTED SATURATION 


\section{APPENDIX}

IOMS RUN: PUREX6

COMMENTS Set 2

DATE: $12 / 7 / 1993-12 / 8 / 93$

TIME: 19:10 PM - 00:15 AM

WASHED SAMPLE:

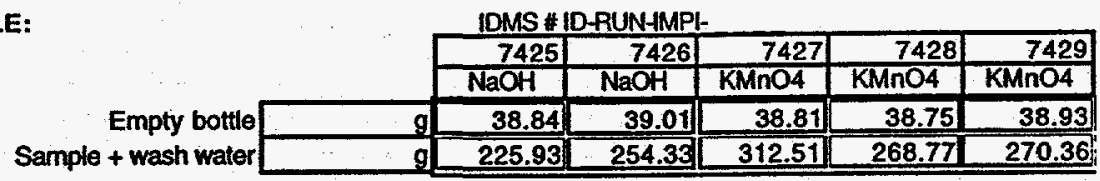

\begin{tabular}{|c|c|c|c|c|c|c|}
\hline t of sample & g & 187.09 & 215.32 & 273.70 & 230.02 & 231.43 \\
\hline $\begin{array}{l}\text { pprox. density of sample } \\
\text { Volume of sample }\end{array}$ & $\frac{g / m !}{m !}$ & $\begin{array}{r}1.1 \\
170.1\end{array}$ & $\begin{array}{r}1.1 \\
195.7\end{array}$ & $\frac{1.1}{248.8}$ & $\frac{1.1}{209.1}$ & $\frac{1.1}{210.4}$ \\
\hline
\end{tabular}

\begin{tabular}{lll|l|l|l|l|}
\hline Conc of $\mathrm{Hg}$ & mg/iter $(\mathrm{ppm})$ & 87.2 & 75.4 & 169.0 & .81 .1 & 62.7 \\
\hline
\end{tabular}

\begin{tabular}{rr|r|r|r|r|r|}
\hline Hg collected & $\mathrm{mg}$ & 14.83 & 14.76 & 42.05 & 16.96 & 13.19 \\
\hline$\%$ of total collected & $\%$ & 14.57 & 14.50 & 41.31 & 16.66 & 12.96 \\
\hline
\end{tabular}

Total Hg collected $\square \quad \mathrm{mg} \quad 101.79$

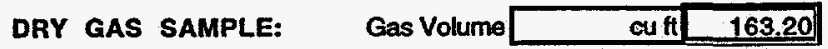

MERCURY CONCENTRATION MEASURED AT FAVC EXIT:

\begin{tabular}{|c|c|c|c|}
\hline & & $9=25^{\circ} \mathrm{C}$ & (e $0^{\circ} \mathrm{C}$ \\
\hline $\mathrm{Hg}$ concentratio & $\mu \mathrm{g} / \mathrm{ft}$ & 623.72 & $680.80 \mu \mathrm{g} / \mathrm{std} \mathrm{ft} 3$ \\
\hline $\mathrm{Hg} \mathrm{O}$ & $\mathrm{g} / \mathrm{m} 3$ & 0.0220 & $0.0240 \mathrm{~g} / \mathrm{std} \mathrm{m3}$ \\
\hline $\begin{array}{l}\text { Hg concentration } \\
\text { Saturation temp }\end{array}$ & $\underset{\mathrm{C}}{\mathrm{mg} / \mathrm{m} 3}$ & 22.0264 & $\begin{array}{r}24.0423 \\
26.27 \\
\text { (by changing) }\end{array}$ \\
\hline
\end{tabular}

MERCURY CONCENTRATION AT SATURATION (PROCESS BASIS, FAVC EXIT) : Average FAVC Exit Temperature C 9.62

$\begin{array}{cc}\mathrm{Hg} \text { concentration } & \mu \mathrm{g} / \mathrm{ft} 3 \\ \mathrm{Hg} \text { concentration } & \mathrm{g} / \mathrm{m} 3 \\ \mathrm{Hg} \text { concentration } & \mathrm{mg} / \mathrm{m} 3\end{array}$

Qavg temp $00^{\circ} \mathrm{C}$

$\mathrm{Hg}$ concentration $\mathrm{mg} / \mathrm{m} 3$

\begin{tabular}{|c|c|}
\hline 151.5 & $156.8 \mu \mathrm{g} / \mathrm{std} \mathrm{ft3}$ \\
\hline 0.0053 & $0.0055 \mathrm{~g} / \mathrm{std} \mathrm{m} 3$ \\
\hline 5.3486 & $5.5369 n$ \\
\hline
\end{tabular}

MEASURED / EXPECTED @ SATURATION 
WSRC-TR-96-352, Rev. 0

\section{APPENDIX}

IDMS RUN: PUREX 6

COMMENTS Set 3

DATE: 12/8/93

TIME: 00:23 AM - 5:30 AM

WASHED SAMPLE:

IDMS \# ID-RUN-IMPI-

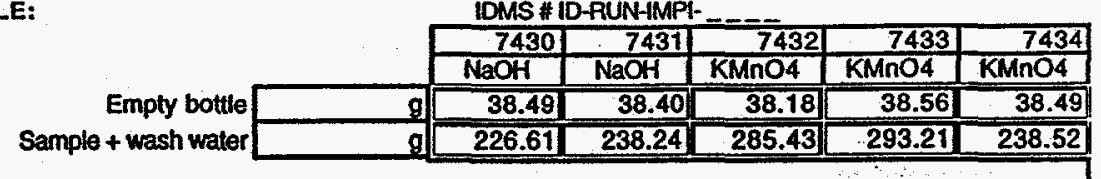

\begin{tabular}{r|r|r|r|r|r|r|} 
Weight of sample & $\mathrm{g}$ & 188.12 & 199.84 & 247.25 & 254.65 & 200.03 \\
\cline { 2 - 7 } & $\mathrm{g} / \mathrm{m} \mid$ & 1.1 & 1.1 & 1.1 & 1.1 & 1.1 \\
\cline { 2 - 7 } & $\mathrm{mpprox}$ density of sample & 171.0 & 181.7 & 224.8 & 231.5 & 181.8 \\
\hline
\end{tabular}

\begin{tabular}{ll|l|l|l|l|l|l|} 
Conc of $\mathrm{Hg}$ & mg/iter (ppm) & 137.0 & 28.7 & 78.9 & & 88.8 & 43.8 \\
\hline
\end{tabular}

\begin{tabular}{rr|r|r|r|r|r|} 
Hg collected & $\mathrm{mg}$ & 23.43 & 5.21 & 17.73 & 20.56 & 7.96 \\
\hline$\%$ of total collected & $\%$ & 31.28 & 6.96 & 23.68 & 27.45 & 10.63 \\
\hline
\end{tabular}

Total $\mathrm{Hg}$ collected $\mathrm{mg} \quad \mathbf{7 4 . 9 0}$

DRY GAS SAMPLE: Gas Volume $\quad$ cut 215.60

MERCURY CONCENTRATION MEASURED AT FAVC EXIT:

\begin{tabular}{|c|c|c|c|}
\hline $\begin{array}{l}\mathrm{Hg} \text { concentration } \\
\mathrm{Hg} \text { concentration }\end{array}$ & $\begin{array}{c}\mu g / f t 3 \\
g / m 3\end{array}$ & $\begin{array}{l}347.40 \\
0.0123\end{array}$ & $\frac{379.20}{0.0134} \mathrm{\mu g} / \mathrm{std} \mathrm{st} 3$ \\
\hline $\begin{array}{l}\mathrm{Hg} \text { concentration } \\
\text { Saturation temp }\end{array}$ & $\underset{\alpha C}{m g / m 3}$ & 12.2684 & $\begin{array}{r}13.3912 \\
19.40 \\
\text { (by changin }\end{array}$ \\
\hline
\end{tabular}

MERCURY CONCENTRATION AT SATURATION (PROCESS BASIS, FAVC EXIT) : Average FAVC Exit Temperature

C

7.42

$\mathrm{Hg}$ concentration

$\mu \mathrm{g} / \mathrm{ft} 3$

$\mathrm{Hg}$ concentration $\quad \mathrm{g} / \mathrm{m} 3$

$\mathrm{Hg}$ concentration $\mathrm{mg} / \mathrm{m3}$

Qavg temp $0.00^{\circ} \mathrm{C}$
\begin{tabular}{|r|r|r|}
\hline 124.0 & 127.4 & $\mu \mathrm{g} / \mathrm{std} \mathrm{ft} 3$ \\
\hline 0.0044 & 0.0045 & $\mathrm{~g} / \mathrm{std} \mathrm{m3}$ \\
\hline 4.3805 & 4.4995 & $\mathrm{mg} / \mathrm{std} \mathrm{m3}$ \\
\hline
\end{tabular}

MEASURED / EXPECTED SATURATION

2.976 


\section{APPENDIX}

IOMS RUN: PUREX6

COMMENTS Set 4

DATE: $12 / 8 / 93$

TIME: 06:15 AM-11:20 AM

WASHED SAMPLE:

IDMS \# ID-RUN-IMPI-

\begin{tabular}{|c|c|c|c|c|c|c|}
\hline & \\
\hline & & 7435 & 7436 & 7437 & 7438 & 7441 \\
\hline & & $\mathrm{NaOH}$ & $\mathrm{NaOH}$ & $\mathrm{KMnO4}$ & $\mathrm{KMnO} 4$ & $\mathrm{KMnO4}$ \\
\hline Empty bottle & $\mathbf{g}$ & 39.08 & 38.46 & 38.20 & 38.65 & 38.15 \\
\hline ample + wash water & $g$ & 220.04 & 276.65 & 264.13 & 337.97 & 218.54 \\
\hline
\end{tabular}

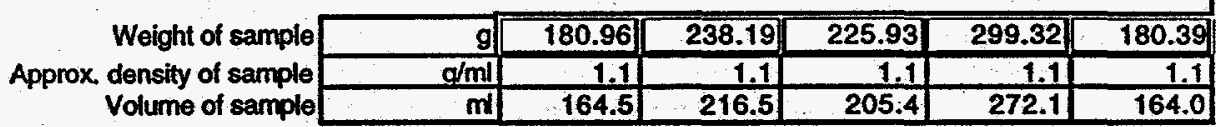

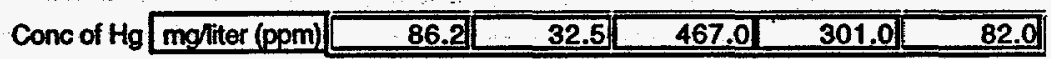

\begin{tabular}{r|r|r|r|r|r|r|} 
Hg collected & $\mathrm{mg}$ & 14.18 & 7.04 & 95.92 & 81.90 & 13.45 \\
\hline$\%$ of total collected & $\%$ & 6.67 & 3.31 & 45.14 & 38.55 & 6.33 \\
\hline
\end{tabular}

Total $\mathrm{Hg}$ collected

$\mathrm{mg} \quad 212.49$

DRY GAS SAMPLE: Gas Volumo cuft 195.00

MERCURY CONCENTRATION MEASURED AT FAVC EXIT:

$\begin{array}{cc}\text { Hg concentration } & \mu \mathrm{g} / \mathrm{ft} 3 \\ \mathrm{Hg} \text { concentration } & \mathrm{g} / \mathrm{m} 3 \\ \mathrm{Hg} \text { concentration } & \mathrm{mg} / \mathrm{m} 3 \\ \text { Saturation temp } & { }^{\mathrm{C}}\end{array}$

\begin{tabular}{|c|c|c|}
\hline $9=25^{\circ} \mathrm{C}$ & Q $0^{\circ} \mathrm{C}$ & \\
\hline 1089.68 & 1189.41 & $\mu \mathrm{g} / \mathrm{std} \mathrm{ft} 3$ \\
\hline 0.0385 & 0.0420 & $g / s t d m 3$ \\
\hline 38.4817 & $\begin{array}{r}42.0036 \\
3313\end{array}$ & $\mathrm{mg} / \mathrm{std} \mathrm{m} 3$ \\
\hline
\end{tabular}

MERCURY CONCENTRATION AT SATURATION (PROCESS BASIS, FAVC EXIT) : Average FAVC Exit Temperature

${ }^{\circ} \mathrm{C}$ $7.86]$

$\begin{array}{lc}\text { Hg concentration } & \mu \mathrm{g} / \mathrm{ft} 3 \\ \mathrm{Hg} \text { concentration } & \mathrm{g} / \mathrm{m} 3 \\ \mathrm{Hg} \text { concentration } & \mathrm{mg} / \mathrm{m} 3\end{array}$

MEASURED / EXPECTED SATURATION

Qavg temp @ $0^{\circ} \mathrm{C}$
\begin{tabular}{|r|r|r|}
\hline 129.1 & 132.8 & $\mathrm{\mu g} / \mathrm{std} \mathrm{ft} 3$ \\
\hline 0.0046 & 0.0047 & $\mathrm{~g} / \mathrm{std} \mathrm{m} 3$ \\
\hline 4.5602 & 4.6914 & $\mathrm{mg} / \mathrm{std} \mathrm{m} 3$
\end{tabular}

Set $=0$

8.953

Sel $=0$

0.000000 


\title{
APPENDIX
}

\author{
IDMS RUN: PUREX 6 \\ COMMENTS Set 5 \\ DATE: 12/8/93 \\ TIME: 11:37 AM-16:35 PM
}

WASHED SAMPLE:

IOMS \# ID-RUN-IMP!-

\begin{tabular}{|c|c|c|c|c|c|c|}
\hline \multirow{3}{*}{\multicolumn{2}{|c|}{ WTLE: }} & \multicolumn{5}{|c|}{$\begin{array}{l}\text { HOMS \# IOA-HONAMIP- } \\
7442\end{array}$} \\
\hline & & 7442 & 7443 & 7444 & 7445 & 7446 \\
\hline & & $\mathrm{NaOH}$ & $\mathrm{NaOH}$ & $\mathrm{KMnO4}$ & $\mathrm{KMnO} 4$ & $\mathrm{KMnO} 4$ \\
\hline Empty bottle & $g$ & 38.15 & 38.82 & 38.87 & 38.47 & 38.77 \\
\hline Sample + wash water & g & 262.54 & 255.35 & 286,40 & 258.90 & 260.55 \\
\hline Weight of sample & g & 224.39 & 216.53 & 247.53 & 220.43 & 221.78 \\
\hline Approx density of sample & $\mathrm{g} / \mathrm{ml}$ & 1.1 & 1.1 & 1.1 & 1.1 & 1.1 \\
\hline Volume of sample & m! & 204.0 & 196.8 & 225.0 & 200.4 & 201.6 \\
\hline Conc of $\mathrm{Hg}$ & mg/titer (ppm) & 83.0 & 22.4 & 391.0 & 14.01 & 1.0 \\
\hline $\mathrm{Hg}$ coll & $\mathbf{m g}$ & 16.931 & 4.409 & 87.986 & 2.805 & 0.204 \\
\hline$\%$ of total & $\%$ & 15.07 & 3.83 & 78.32 & 2.501 & 0.18 \\
\hline
\end{tabular}

Total Hg collected $\quad \mathrm{mg} \quad 112.34$

DRY GAS SAMPLE: Gas Volumo $\quad$ cuft 163.50

MERCURY CONCENTRATION MEASURED AT FAVC EXIT:

$\mathrm{Hg}$ concentration

Hg concentration

Hg concentration

Saturation temp

$$
\underset{\substack{\mu g / t t 3 \\ g / m 3 \\ \mathrm{mg} / \mathrm{m} 3}}{{ }_{\mathrm{C}}}
$$

\begin{tabular}{|c|c|}
\hline$\frac{Q=25^{\circ} \mathrm{C}}{687.07}$ & $\frac{0^{\circ} \mathrm{C}}{749.95} \mu \mathrm{g} / \mathrm{std} \mathrm{ft} 3$ \\
\hline 0.0243 & $0.0265 \mathrm{~g} / \mathrm{st}$ \\
\hline 24.2635 & $\frac{26.4842}{27.43} \mathrm{mg} / \mathrm{std} \mathrm{m} 3$ \\
\hline
\end{tabular}

MERCURY CONCENTRATION AT SATURATION (PROCESS BASIS, FAVC EXIT) : Average FAVC Exit Temperature

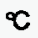

$$
\begin{array}{lc}
\mathrm{Hg} \text { concentration } & \mu \mathrm{g} / \mathrm{hl} 3 \\
\mathrm{Hg} \text { concentration } & \mathrm{g} / \mathrm{m} 3 \\
\mathrm{Hg} \text { concentration } & \mathrm{mg} / \mathrm{m} 3
\end{array}
$$

MEASURED / EXPECTED saturation

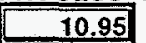

Cavg temp $0^{\circ} \mathrm{C}$

$170.6 \quad 177.5 \mu \mathrm{g} / \mathrm{std} \mathrm{tt3}$ \begin{tabular}{l|l|l|l|l}
0.0060 & 0.0063 & glstd m3
\end{tabular} $\begin{array}{lll}6.0252 & 6.2668 & \mathrm{mg} / \mathrm{std} \mathrm{m3}\end{array}$

Set=0

0.000000 


\section{APPENDIX}

IDMSRUN: BLI

COMMENTS FORMICACID ADDTION FAVCIN

DATE: $6 / 20 / 90$

TIME: 16:10-17:15

WASHED SAMPLE:

IDMS\# ID-RUN-IMPI-

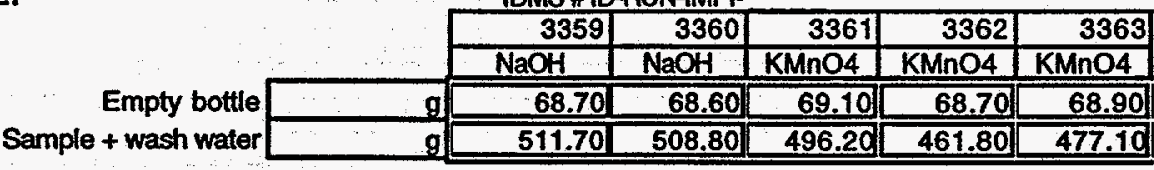

\begin{tabular}{|r|r|r|r|r|r|r|} 
Weight of sample & $g$ & 427.3 & 414.1 & 545.3 & 480.7 & 358.9 \\
\cline { 2 - 7 } Approx. density of sample & $\mathrm{g} / \mathrm{m}$ & 1.10 & 1.10 & 1.10 & 1.10 & 1.10 \\
\hline Volume of sample & $\mathrm{m}$ & 388.5 & 376.5 & 495.7 & 437.0 & 326.3 \\
\cline { 2 - 7 } & & &
\end{tabular}

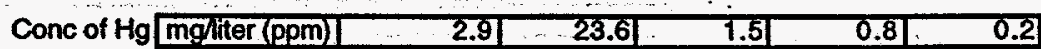

\begin{tabular}{rr|r|r|r|r|r|}
$\mathrm{Hg}$ collected & $\mathrm{mg}$ & 1.1 & 8.9 & 0.7 & 0.3 & 0.1 \\
\hline
\end{tabular}

Total Hg collected $\quad \mathrm{mg} \quad 11.2$

DRY GAS SAMPLE: Gas Volume $\quad$ cu ft $\quad 6.80$

MERCURY CONCENTRATION MEASURED AT FAVC INLET:

Hg concentration

$\mathrm{Hg}$ concentration

$\mathrm{Hg}$ concentration

$\mu g / f t 3$

$\mathrm{g} / \mathrm{m} 3$

$\mathrm{mg} / \mathrm{m} 3$

Saturation temp

c

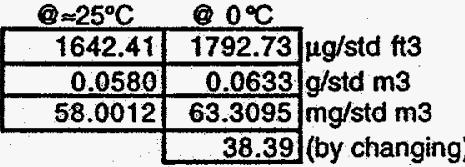

MERCURY CONCENTRATION AT SRATC EXIT CALCULATED FROM MEASURED FAVC INLET:

$\begin{array}{lr}\mathrm{Hg} \text { concentration } & \mu \mathrm{g} / \mathrm{tt} 3 \\ \mathrm{Hg} \text { concentration } & \mathrm{g} / \mathrm{m} 3 \\ \mathrm{Hg} \text { concentration } & \mathrm{mg} / \mathrm{m} 3\end{array}$

\begin{tabular}{|c|c|}
\hline \#DIV/O! & \\
\hline \#DIV/0! & \#DIV/O! \\
\hline
\end{tabular}

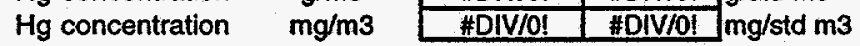

MERCURY CONCENTRATION AT SATURATION (PROCESS BASIS, SRATC EXIT) :

Average SRATC Exit Temperature

C 43.97

Hg concentration $\mathrm{Hg}$ concentration

$\mu \mathrm{g} / \mathrm{ft} 3$

$\mathrm{g} / \mathrm{m} 3$

$\mathrm{Hg}$ concentration $\mathrm{mg} / \mathrm{m} 3$

\begin{tabular}{|r|r|r|}
\hline avg lemp & $000^{\circ} \mathrm{C}$ & \\
\hline 2350.4 & 2728.7 & $\mu \mathrm{g} / \mathrm{std} \mathrm{ft} 3$ \\
\hline 0.0830 & 0.0964 & $\mathrm{~g} / \mathrm{std} \mathrm{m3}$ \\
\hline 83.0029 & 96.3637 & $\mathrm{mg} / \mathrm{std} \mathrm{m3}$
\end{tabular}

MERCURY CONCENTRATION AT FAVC INLET

SRAT/FAVC flow ratio

FAVC Inlet Temperature

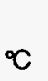

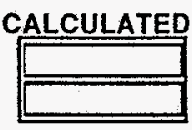

$\mathrm{Hg}$ concentration

Hg concentration

$\mu \mathrm{g} / \mathrm{ft} 3$

$g / m 3$

$\mathrm{Hg}$ concentration

$\mathrm{mg} / \mathrm{m} 3$

\begin{tabular}{|c|c|}
\hline QFAVC $\ln T$ & (Q) $0^{\circ} \mathrm{C}$ \\
\hline 0.0 & \multirow{2}{*}{0.00} \\
\hline 0.0000 & \\
\hline 0.0000 & 0.0000 \\
\hline
\end{tabular}

MEASURED / EXPECTED @ SATURATION

\#DIV/O! 
WSRC-TR-96-352, Rev. 0

\section{APPENDIX}

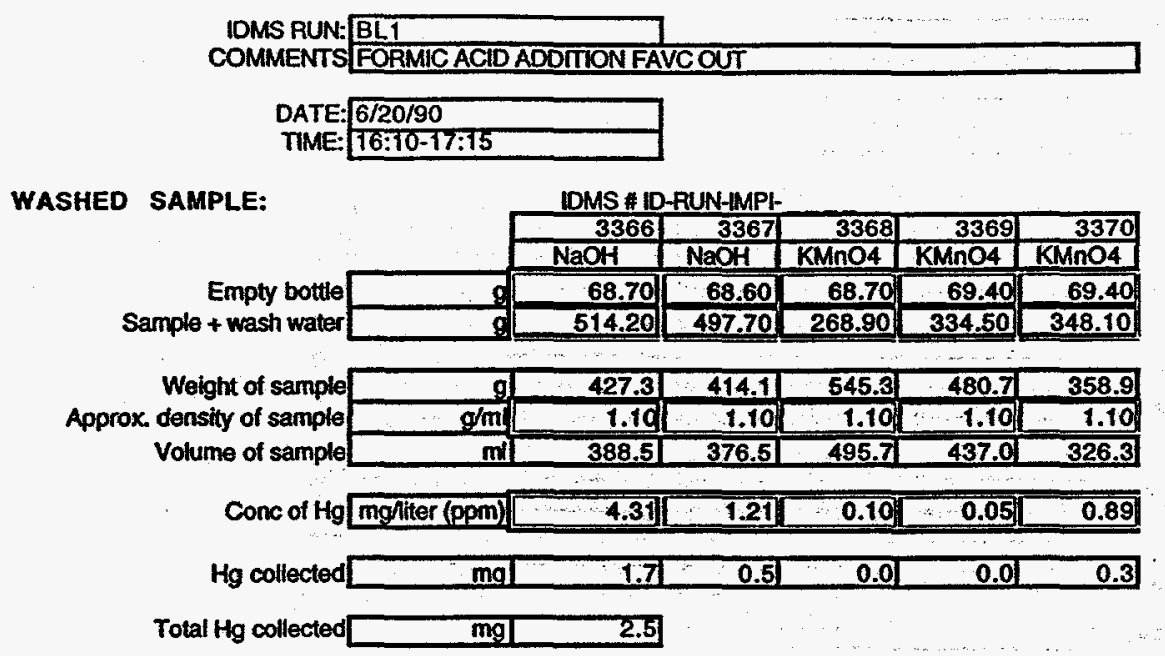

DRY GAS SAMPLE:

Gas Volume $\square$ cuf $\quad 6.30$

MEASURED MERCURY CONCENTRATION:

\begin{tabular}{|c|c|c|c|}
\hline $\begin{array}{l}\mathrm{Hg} \text { concentration } \\
\mathrm{Hg} \text { concentration }\end{array}$ & $\frac{\mathrm{ug} / \mathrm{ft3}}{\mathrm{g} / \mathrm{m3}}$ & $\frac{395.48}{0.0140}$ & \\
\hline Hg concentration & $\mathrm{mg} / \mathrm{m3}$ & 13.97 & \\
\hline $\mathrm{Hg}$ concentration & pem by vol & $\frac{1.678}{2177}$ & (assuming 1 atm pressure) \\
\hline $\begin{array}{l}\text { Saturation temp } \\
\text { Subcooling of } \mathrm{Hg}\end{array}$ & $\frac{c}{c}$ & $\frac{20.72}{0.21}$ & \\
\hline
\end{tabular}

MERCURY CONCENTRATION AT SATURATION (PROCESS BASIS) :

Average Temperature $\square \quad 20.51$

$\mathrm{Hg}$ concentration $\quad \mathrm{ug} / \mathrm{K} 3 \mathrm{3}$

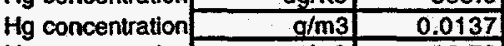

Hg concentration $\quad \mathrm{mg} / \mathrm{m} 3 \quad 13.72$

$\mathrm{Hg}$ concentration Dom by yol $\quad 1.648$ 


\section{APPENDIX}

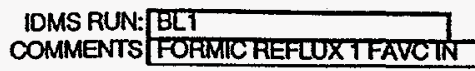

DATE: 6/20/90

TIME: $22: 00-23700$

WASHED SAMPLE:

IDMS \# ID-RUNAIMPI-

\begin{tabular}{|c|c|c|c|c|c|c|}
\hline & \multicolumn{4}{|c|}{3379} & \\
\hline & & $\mathrm{NaOH}$ & $\mathrm{NaOH}$ & KMnO4 & $\mathrm{KMnO4}$ & KMnO4 \\
\hline Empty bottle & g & 68.80 & 68.40 & 69.80 & 69.00 & 68.6 \\
\hline ample + wash water & g & 662.70 & 438.60 & 303.00 & 538.20 & 404.30 \\
\hline
\end{tabular}

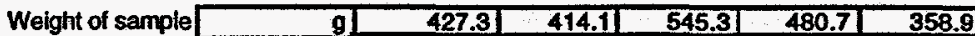
Approx. density of sample

Volume of sample

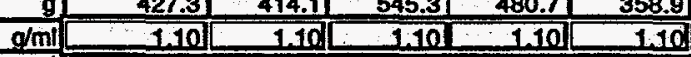

\begin{tabular}{llllll}
\hline$m \mid$ & 3888.5 & 376.5 & 495.7 & 437.0 & 326.3 \\
\hline
\end{tabular}

\begin{tabular}{l|l|l|l|l|l|} 
Conc of $\mathrm{Hg}$ mg/ter $(\mathrm{ppm})$ & 31.30 & 4.30 & 39.00 & 0.26 & 0.05 \\
\hline
\end{tabular}

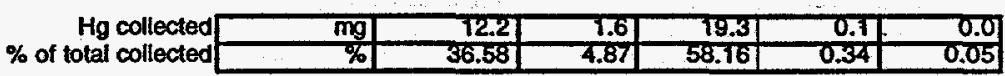

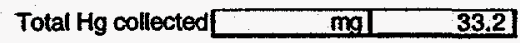

DRY GAS SAMPLE: Gas Volume $\quad$ cu ftr 37.

MERCURY CONCENTRATION MEASUREO AT FAVC INLET:

\begin{tabular}{|c|c|c|c|c|}
\hline \multirow{3}{*}{$\begin{array}{l}\text { Hg concentration } \\
\mathrm{Hg} \text { concentration } \\
\text { Hg concentration } \\
\text { Saturation temp }\end{array}$} & \multirow{3}{*}{$\begin{array}{c}\mu g / f t 3 \\
g / m 3 \\
m g / m 3 \\
{ }_{C}\end{array}$} & \multicolumn{3}{|c|}{ 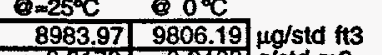 } \\
\hline & & 0.3773 & 0.3463 & $g / s t d ~ m 3$ \\
\hline & & 317.2658 & $\begin{array}{r}346.3024 \\
62.30 \\
\end{array}$ & $\begin{array}{l}\mathrm{mg} / \mathrm{std} \mathrm{m} 3 \\
\text { (by changin }\end{array}$ \\
\hline
\end{tabular}

MERCURY CONCENTRATION AT SRATC EXIT CALCULATED FROM MEASURED FAVC INLET:

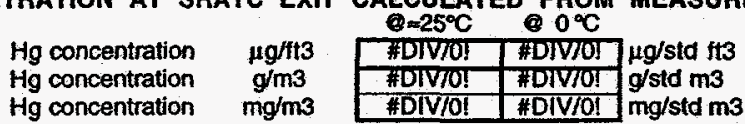

MERCURY CONCENTRATION AT SATURATION (PROCESS BASIS, SRATC EXIT) :

Average SRATC Exit Temperature

Hg concentration
Hg concentration

$\stackrel{\mu g / t+3}{g / m 3}$

$\mathrm{mg} / \mathrm{ms}$
${ }^{\circ} \quad 36.80$

\begin{tabular}{|c|c|}
\hline avg gemp & 80 \\
\hline$\frac{1091.0}{0.0494}$ & $\frac{1585.9}{0.0560}$ \\
\hline & \\
\hline
\end{tabular}

MERCURY CONCENTRATION AT FAVC INLET CALCULATED FROM SATURATION:

SRAT/FAVC flow ratio

FAVC inlet Temperature

c

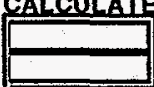

Hg concentration $\mu g / f t 3$

$\mathrm{Hg}$ concentration $\mathrm{g} / \mathrm{m} 3$

$\mathrm{Hg}$ concentration $\mathrm{mg} / \mathrm{m} 3$

MEASURED / EXPECTED Q SATURATION

\begin{tabular}{|c|c|}
\hline$\frac{\text { OFAVCln }}{0.0}$ & \multirow{2}{*}{ Tomom } \\
\hline 0.0000 & \\
\hline & 0.0000 \\
\hline
\end{tabular}

\#DIV/0! 


\section{APPENDIX}

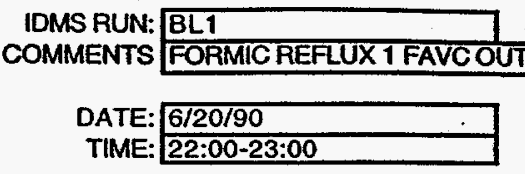

WASHED SAMPLE:

\begin{tabular}{|c|c|c|c|c|c|c|}
\hline HED SAMPLE: & & DMS \# ID & ZUN-IMPF- & & & \\
\hline & & 3385 & 3386 & 3387 & 3388 & 3389 \\
\hline & & $\mathrm{NaOH}$ & $\mathrm{NaOH}$ & KMnO4 & KMnO4 & $\mathrm{KMnO} 4$ \\
\hline Empty bottle & g & 69.00 & 68.70 & 69.30 & 68.90 & 68.90 \\
\hline Sample + wash water & g & 490.90 & 492.10 & 565.80 & 533.60 & 301.20 \\
\hline Weight of sample & g] & 421.90 & 423.40 & 496.50 & 464.70 & 232.30 \\
\hline Approx. density of sample & $\mathrm{g} / \mathrm{m} \mathbf{1}$ & 1.1 & 1.1 & 1.1 & 1.1 & 1.1 \\
\hline Volume of sample & $\mathbf{m}$ & 383.5 & 384.9 & 451.4 & 422.5 & 211.2 \\
\hline Conc of $\mathrm{Hg}$ & mgliter (ppm) & 13.59 & 2.20 & 17.10 & 0.11 & $\ldots 0.04$ \\
\hline $\mathrm{Hg}$ collected & $\mathrm{mg}$ & 5.212 & 0.847 & 7.718 & 0.046 & 0.008 \\
\hline$\%$ of total collected & $\%$ & 37.68 & 6.12 & 55.80 & 0.34 & 0.06 \\
\hline
\end{tabular}

DRY GAS SAMPLE:

Gas Volume $\quad$ cu $\mathrm{tt} \quad 6.30$

MEASURED MERCURY CONCENTRATION:

\begin{tabular}{|c|c|c|c|}
\hline Hg concentration & $\mathrm{ug} / \mathrm{ft3}$ & 2195.62 & \\
\hline $\mathrm{Hg}$ concentration & $\mathrm{g} / \mathrm{m} 3$ & 0.0775 & \\
\hline Hg concentration & $\mathrm{mg} / \mathrm{m} 3$ & 77.54 & \\
\hline $\begin{array}{l}\mathrm{Hg} \text { concentration } \\
\text { Saturation temo }\end{array}$ & ppm by vol & $\frac{9.015}{43.01}$ & (assuming 1 alm pressure) \\
\hline Subcooling of $\mathrm{Hg}$ & ${ }^{\circ} \mathrm{C}$ & 31.94 & \\
\hline
\end{tabular}

MERCURY CONCENTRATION AT SATURATION (PROCESS BASIS) :

Average Temperature 11.07 estimated

\begin{tabular}{l|r|r|} 
Hg concentration & $\mathrm{ug} / \mathrm{ft} 3$ & 172.4 \\
\cline { 2 - 3 } $\mathrm{Hg}$ concentration & $\mathrm{g} / \mathrm{m} 3$ & 0.0061 \\
\cline { 2 - 3 } $\mathrm{Hg}$ concentration & $\mathrm{mg} / \mathrm{m} 3$ & 6.09 \\
Hg concentration & opm by vol & 0.708 \\
\hline
\end{tabular}




\section{APPENDIX}

IDMS RUN: BLI
COMMENTS FORMICREFLUX2FAVCIN

DATE: 6/21/90

TIME: 01:00-02:00

WASHED SAMPLE:

IDMS : ID-RUN-IMPI

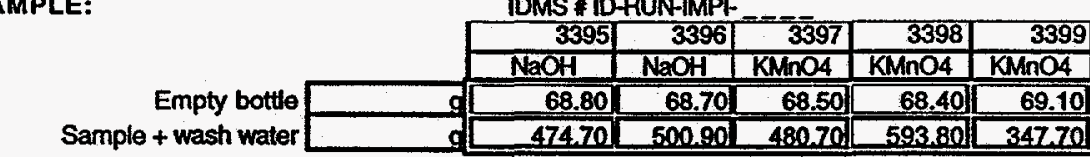

\begin{tabular}{|c|c|c|c|c|c|c|}
\hline Weight of sample & g & 427.31 & 414.1 & 545.3 & 480.7 & 358.9 \\
\hline Approx. density of sample & $\mathrm{g} / \mathrm{ml}$ & 1.10 & 1.10 & 1.10 & 1.10 & 1.10 \\
\hline Volume of sample & $m$ & 388.5 & 376.5 & 495.7 & 437.0 & 326.3 \\
\hline Cone of $\mathrm{Hg}$ & pom) & 5.80 & 101 & 26,40 & 143 & 0.55 \\
\hline
\end{tabular}

\begin{tabular}{rr|r|r|r|r|r|} 
Hg collected & $\mathrm{mg}$ & 2.3 & 0.4 & 13.1 & 0.6 & 0.2 \\
\hline \% of total collected & \% & 13.63 & 2.30 & 79.20 & 3.78 & 1.09 \\
\hline
\end{tabular}

Total Hg collected $\square \quad \mathrm{mg} / \mathbf{1 6 . 5}$

DRY GAS SAMPLE: Gas Volume $\quad \mathrm{cuft} r 5.10$

MERCURY CONCENTRATION MEASURED AT FAVC INLET:

\begin{tabular}{|c|c|c|c|}
\hline $\begin{array}{l}\mathrm{Hg} \text { concentration } \\
\mathrm{Hg} \text { concentration } \\
\mathrm{Hg} \text { concentration } \\
\text { Saturation temp }\end{array}$ & $\underset{\substack{\mu g / f t 3 \\
g / m 3 \\
m g / m 3}}{ }$ & $\begin{array}{r}3240.16 \\
0.1144 \\
114.4252 \\
\end{array}$ & $\begin{array}{r}3536.70 \\
0.1249 \\
124.8975 \\
47.52\end{array}$ \\
\hline
\end{tabular}

MERCURY CONCENTRATION AT SRATC EXIT CALCULATED FROM MEASURED FAVC INLET:

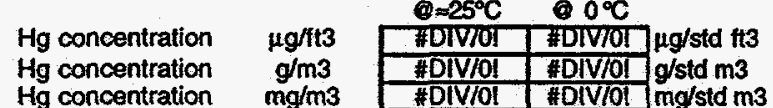

MERCURY CONCENTRATION AT SATURATION (PBOCESS BASIS, SRATC EXIT) : Average SRATC Exit Temperature

$$
{ }^{\circ} \quad 30.77
$$

\begin{tabular}{|c|c|c|c|}
\hline & & Qavg temp & (2) $0^{\circ} \mathrm{C}$ \\
\hline & $\begin{array}{c}\mu \mathrm{g} / \mathrm{ft3} \\
\mathrm{g} / \mathrm{m} 3 \\
\mathrm{mg} / \mathrm{m} 3\end{array}$ & $\begin{array}{r}884.9 \\
0.0312 \\
31.2493 \\
\end{array}$ & 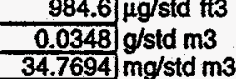 \\
\hline
\end{tabular}

MERCURY CONCENTRATION AT FAVC INLET CALCULATED FROM SATURATION: SRAT/FAVC flow ratio

FAVC Inlet Temperature

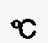

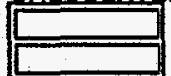

Hg concentration Hg concentration

$\mathrm{Hg}$ concentration

$$
\underset{\mathrm{gg} / \mathrm{ft} 3}{\mathrm{~m} / \mathrm{m} 3}
$$

\begin{tabular}{|c|c|}
\hline & \\
\hline 0.0 & $0.0 \mu$ \\
\hline $\begin{array}{l}0.0000 \\
0.0000\end{array}$ & 0.00000 \\
\hline
\end{tabular}

MEASURED / EXPECTED SATURATION

\#DIV/O! 


\section{APPENDIX}

IDMS RUN: BL1

COMMENTS FORMIC REFLUX 2 FAVC OUT

DATE: $6 / 21 / 90$

TIME: 01:00-02:00

WASHED SAMPLE:

IDMS \# ID-RUN-IMPI-

\begin{tabular}{|c|c|c|c|c|c|c|}
\hline & & 3402 & 3403 & 3404 & 3405 & 3406 \\
\hline & & $\mathrm{NaOH}$ & $\mathrm{NaOH}$ & $\mathrm{KMnO} 4$ & $\mathrm{KMnO4}$ & KMnO4 \\
\hline Empty bottle & $\mathbf{g}$ & 68.90 & 68.40 & 68.60 & 68.20 & 68.60 \\
\hline ample + wash water & 9 & 496.20 & 482.50 & 613.90 & 548.90 & 427.50 \\
\hline
\end{tabular}

\begin{tabular}{rl|r|r|r|r|r|} 
Weight of sample & $g$ & 427.3 & 414.1 & 545.3 & 480.7 & 358.9 \\
\cline { 2 - 6 } Approx. density of sample & $g / \mathrm{mi}$ & 1.10 & 1.10 & 1.10 & 1.10 & 1.10 \\
\cline { 2 - 6 } Volume of sample & $\mathrm{m}$ & 388.5 & 376.5 & 495.7 & 437.0 & 326.3 \\
\hline
\end{tabular}

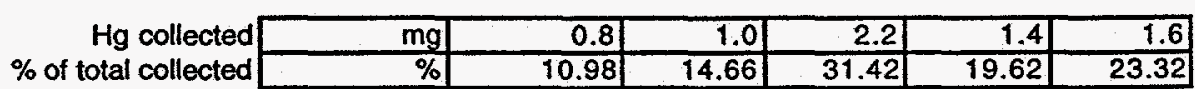

Total $\mathrm{Hg}$ collected $\quad \mathrm{mg} \quad 7.0$

DRY GAS SAMPLE:

Gas Volume $\quad$ cuff $\quad 6.90$

MEASURED MERCURY CONCENTRATION:

\begin{tabular}{rr}
$\mathrm{Hg}$ concentration & $\mathrm{ug} / \mathrm{ft} 3$ \\
\hline & 1019.93 \\
\hline
\end{tabular}

\begin{tabular}{rrr}
$\mathrm{Hg}$ concentration & $\mathrm{g} / \mathrm{m} 3$ & 0.0360 \\
\hline
\end{tabular}

\begin{tabular}{rr}
$\mathrm{Hg}$ concentration & $\mathrm{mg} / \mathrm{m} 3$ \\
\hline
\end{tabular}

Hg concentration $\quad$ ppm by vol $\quad 4.152$ (assuming 1 atm pressure)

Saturation temp

Subcooling of $\mathrm{Hg}$

c. 32.90

25

MERCURY CONCENTRATION AT SATURATION (PROCESS BASIS), :

\begin{tabular}{|c|c|c|}
\hline Average Temperature & ${ }^{\circ}$ & 8.65 \\
\hline $\mathrm{Hg}$ concentration & $\mathrm{ug} / \mathrm{ft3}$ & 138.7 \\
\hline $\mathrm{Hg}$ concentration & $\mathrm{g} / \mathrm{m} 3$ & 0.0049 \\
\hline $\mathrm{Hg}$ concentration & $m g / m 3$ & 4.90 \\
\hline $\mathrm{Hg}$ concentration & ppm by vol & 0.565 \\
\hline
\end{tabular}

